\title{
Comparative safety of anti-epileptic drugs during pregnancy: a systematic review and network meta-analysis of congenital malformations and prenatal outcomes
}

Areti Angeliki Veroniki ${ }^{1}$, Elise Cogo ${ }^{1}$, Patricia Rios ${ }^{1}$, Sharon E. Straus ${ }^{1,2}$, Yaron Finkelstein ${ }^{3,4,5}$, Ryan Kealey ${ }^{1}$, Emily Reynen ${ }^{1}$, Charlene Soobiah ${ }^{1,6}$, Kednapa Thavorn ${ }^{7,8,9}$, Brian Hutton ${ }^{7,10}$, Brenda R. Hemmelgarn ${ }^{11}$,

Fatemeh Yazdi ${ }^{1}$, Jennifer D'Souza', Heather MacDonald ${ }^{1}$ and Andrea C. Tricco ${ }^{1,12^{*}}$

\begin{abstract}
Background: Pregnant women with epilepsy frequently experience seizures related to pregnancy complications and are often prescribed anti-epileptic drugs (AEDs) to manage their symptoms. However, less is known about the comparative safety of AED exposure in utero. We aimed to compare the risk of congenital malformations (CMs) and prenatal outcomes of AEDs in infants/children who were exposed to AEDs in utero through a systematic review and Bayesian random-effects network meta-analysis.

Methods: MEDLINE, EMBASE, and Cochrane CENTRAL were searched from inception to December 15, 2015. Two reviewers independently screened titles/abstracts and full-text papers for experimental and observational studies comparing mono- or poly-therapy AEDs versus control (no AED exposure) or other AEDs, then abstracted data and appraised the risk of bias. The primary outcome was incidence of major CMs, overall and by specific type (cardiac malformations, hypospadias, cleft lip and/or palate, club foot, inguinal hernia, and undescended testes).
\end{abstract}

Results: After screening 5305 titles and abstracts, 642 potentially relevant full-text articles, and 17 studies from scanning reference lists, 96 studies were eligible ( $n=58,461$ patients). Across all major $C M s$, many AEDs were associated with higher risk compared to control. For major CMs, ethosuximide (OR, 3.04; 95\% Crl, 1.23-7.07), valproate $(\mathrm{OR}, 2.93 ; 95 \% \mathrm{Crl}, 2.36-3.69)$, topiramate $(\mathrm{OR}, 1.90 ; 95 \% \mathrm{Crl}, 1.17-2.97)$, phenobarbital $(\mathrm{OR}, 1.83 ; 95 \% \mathrm{Crl}, 1$. 35-2.47), phenytoin (OR, 1.67; 95\% Crl, 1.30-2.17), carbamazepine (OR, 1.37; 95\% Crl, 1.10-1.71), and 11 polytherapies were significantly more harmful than control, but lamotrigine $(\mathrm{OR}, 0.96 ; 95 \% \mathrm{Crl}, 0.72-1.25)$ and levetiracetam $(\mathrm{OR}, 0$. 72; 95\% Crl, 0.43-1.16) were not.

(Continued on next page)

\footnotetext{
* Correspondence: triccoa@smh.ca

${ }^{1}$ Knowledge Translation Program, Li Ka Shing Knowledge Institute, St.

Michael's Hospital, 209 Victoria Street, East Building, Toronto, Ontario M5B 1W8, Canada

${ }^{12}$ Epidemiology Division, Dalla Lana School of Public Health, University of Toronto, 6th Floor, 155 College Street, Toronto, Ontario M5T 3M7, Canada Full list of author information is available at the end of the article
} 
(Continued from previous page)

Conclusion: The newer generation AEDs, lamotrigine and levetiracetam, were not associated with significant increased risks of CMs compared to control, and were significantly less likely to be associated with children experiencing cardiac malformations than control. However, this does not mean that these agents are not harmful to infants/children exposed in utero. Counselling is advised concerning teratogenic risks when the prescription is written for a woman of childbearing age and before women continue with these agents when considering pregnancy, such as switching from polytherapy to monotherapy with evidence of lower risk and avoiding AEDs, such as valproate, that are consistently associated with CMs. These decisions must be balanced against the need for seizure control.

\section{Systematic Review Registration: PROSPERO CRD42014008925}

Keywords: Network meta-analysis, Systematic review, Epilepsy, Fetus, Pregnancy, Adverse effects, Antiepileptic drugs, Congenital malformations, Miscarriage, Knowledge synthesis

\section{Background}

Epilepsy, the most common chronic neurological condition, affects $0.6-1 \%$ of the population [1, 2]. Epilepsy in pregnant women causes frequent seizures, increasing the risk of pregnancy-related complications [3, 4]. Antiepileptic drugs (AEDs) are prescribed to reduce the severity of epilepsy or help manage other conditions such as pain, psychiatric disorders, and migraine [5]. Women taking AEDs have a greater risk of miscarriage and teratogenicity, including a $4-8 \%$ chance of giving birth to a child with a major congenital malformation (CM), because these agents can be transferred to the fetus via the placenta $[3,4,6-8]$. Since the first documentation of teratogenicity of AEDs in the 1960s $[9,10]$, the use of many first-generation AEDs (e.g., valproate) in pregnant women with epilepsy has been studied extensively. Several large-scale pregnancy registries were established to evaluate the safety of first- and newer-generation (e.g., gabapentin) AEDs [11, 12]. However, little is known about the "comparative" safety of AED exposure in utero, and previous studies comparing multiple AEDs are often small and underpowered. As such, we compared the safety of AEDs in infants and children exposed in utero through a systematic review and network metaanalysis (NMA).

\section{Methods}

Our protocol was registered with PROSPERO (CRD 42014008925) and published in an open-access journal (Additional file 1) [13]. Our NMA conforms to the ISPOR [14] guidance and PRISMA-NMA (Additional file 2) [15].

\section{Eligibility criteria}

Pregnant women taking AEDs for any indication were eligible. Studies reporting on the following AEDs as monotherapy or polytherapy of any dose were included: first-generation (carbamazepine, clobazam, clonazepam, ethosuximide, phenobarbital, phenytoin, primidone, valproate) and newer-generation (marketed after 1990; gabapentin, lamotrigine, levetiracetam, oxcarbazepine, topiramate, vigabatrin). The comparators were placebo, no AED treatment (women not exposed to AED but with the same indications for their use), or other AEDs alone or in combination. Papers judged to include data from the same patients were excluded from the analysis to avoid double-counting. Companion reports of included studies were used for supplementary information only.

The primary outcomes were the incidence of overall and specific types of major $\mathrm{CM}$, which were defined as malformations present from birth with surgical, medical, functional, or cosmetic importance [16]. When studies also reported on major $\mathrm{CM}$ cases that were diagnosed prenatally and resulted in elective terminations, these were included in the CM analysis. For specific CM types, the six most frequently occurring in the literature were selected, namely cardiac, cleft lip/palate, club foot, hypospadias, inguinal hernia, and undescended testes (boys only). The secondary outcomes of interest were the incidence of combined fetal losses, prenatal growth retardation, preterm birth, and minor CMs (i.e., any CM that did not qualify as a major CM; Additional file 3: Appendix A). The "combined" fetal loss types outcome includes total fetal losses reported as well as studies that only report on one type of fetal loss (e.g., stillbirths). Randomized clinical trials (RCTs), quasi-RCTs, and observational studies with a control group examining the effects of AEDs on infants and children ( $\leq 12$ years of age) who were exposed to AEDs in utero were included. No language or other restrictions were employed.

\section{Information sources}

An experienced librarian developed the search strategies in MEDLINE, EMBASE, and the Cochrane CENTRAL Register of Controlled Trials. The MEDLINE search strategy was peer-reviewed by another librarian using the Peer Review of Electronic Search Strategies checklist [17], and the final version is provided in our protocol 
[13]. The literature search was initially conducted from inception until March 18, 2014, and a rapid update was conducted on December 15, 2015. Reference lists of all included studies and relevant reviews were scanned. Unpublished studies were sought by locating relevant conference abstracts and contacting authors of included studies and AED manufacturers.

\section{Study selection and data collection}

After the team conducted two pilot-tests of the eligibility criteria among 10 reviewers (12\% disagreements), pairs of reviewers screened each title/abstract independently and conflicts $(6 \%)$ were resolved through discussion. Subsequently, three level 2 screening pilots (26\% disagreements) occurred, as well as three data abstraction pilots. The same process was followed for potentially relevant full-text articles (16\% conflicts) and data abstraction. Authors were contacted for studies published in the last 10 years to clarify unclear or missing data.

The 'no AED use' arms were only included if the control group had the same indication as the active arm in the study (e.g., both had epilepsy). The malformation rates were expressed on a basis of livebirths plus stillbirths, based on the number of pregnant women enrolled in the study.

\section{Appraisal of methodological quality and risk-of-bias}

Two reviewers independently appraised quality using the Cochrane risk-of-bias tool [18] and Newcastle-Ottawa Scale [19]. The comparison-adjusted funnel plot was used to assess publication bias and small-study effects for outcomes including at least 10 studies [20].

In the comparison-adjusted funnel plot, the overall treatment effect for each comparison was estimated under the fixed-effect meta-analysis model and its difference from the study-specific treatment effect versus the study-specific standard error was plotted. All AEDs were ordered from oldest to newest according to their international market approval date. The comparison-adjusted funnel plot does not account for correlations induced by multi-arm trials, which may possibly cause overestimation and mask funnel plot asymmetry. To surmount most correlations in multi-arm trials, only data points corresponding to the study-specific basic parameters (treatment comparisons with common comparator) were plotted. For this, the control group was considered the common comparator or, if this was missing, the oldest treatment comparator was used against the remaining AEDs of the corresponding study.

\section{Synthesis of included studies}

A random-effects meta-analysis model was applied because the studies differed methodologically and clinically. Outcome data were pooled using the odds ratio
(OR) and, for two or more studies, the OR was estimated using Bayesian hierarchical models and a Markov Chain Monte Carlo algorithm. When treatment comparisons formed a connected network of evidence, a random-effects NMA was conducted [21] using treatment nodes pre-specified by the team. Multiple doses were combined in nodes, because this information was not reported consistently across the studies. In both pairwise meta-analyses and NMAs, we assumed common within-network between-study variance $\left(\tau^{2}\right)$ across treatment comparisons, since there were many treatment comparisons, including a single study where the $\left(\tau^{2}\right)$ was not estimable.

Prior to applying a NMA, the transitivity assumption was assessed using age, baseline risk, treatment indication, timing of exposure, and risk-of-bias as potential treatment effect modifiers. The mean of each continuous potential effect modifier and the mode (i.e., most frequent value) of each categorical potential effect modifier for each pairwise comparison and outcome were presented in tables [22]. For each outcome, the entire network was evaluated for inconsistency using the design-by-treatment interaction model [23, 24]. The random-effects model was used when multiple studies were available in each design in the network; alternatively, we applied the fixed-effect model. If the global test suggested inconsistency, local inconsistency in specific network paths was assessed using the loop-specific method assuming common within-loop $\tau^{2}[25,26]$. This was a clinically reasonable assumption, since the treatments were of the same nature. When statistically significant inconsistency or important heterogeneity were detected, the data was checked for errors. If no errors were identified, network meta-regression, subgroup, or sensitivity analyses were conducted. For the overall major $\mathrm{CM}$, combined fetal losses, and prenatal growth outcomes, network meta-regression were performed for age and baseline risk (i.e., using the control group), assuming a common fixed coefficient across comparisons. For these outcomes, a subgroup analysis was conducted for AED generation (i.e., older AEDs versus newer generation $A E D s$ ), and study designs (i.e., observational versus RCTs). Sensitivity analyses were conducted on the same outcomes restricting to studies with treatment indication (i.e., including only women with epilepsy), timing of at least first trimester exposure, large study size (i.e., > 300 patients), maternal alcohol intake, and higher methodological quality using two items of the Newcastle-Ottawa Scale for cohort studies (adequacy of follow-up of cohorts, comparability of cohorts) and low overall risk-of-bias for RCTs (component approach using randomization and allocation concealment items) [27]. For the overall major CM outcome, sensitivity analyses were conducted for cohort studies, 
folic acid used by more than $50 \%$ of women and family history of major CMs, including a large international registry study (EURAP) $[28,29]$ that was not included in the primary analysis due to potential partial overlap of participants with other studies and removing three potentially overlapping studies from Australia, Spain, and Argentina [30-32]. For combined fetal losses and prenatal growth outcomes, sensitivity analysis was conducted for maternal tobacco use. Finally, for the overall major CM, combined fetal losses, and prenatal growth outcomes, the model suggested by Schmitz et al. [33] for different study designs was applied.

In the Schmitz et al. [33] model, bias adjustment to account for over-precision or for over/under-estimation was not introduced, as we were uncertain about the magnitude of bias that might have been introduced from including the observational studies. The goodness-of-fit was measured using the posterior mean of the residual deviance, the degree of between-study heterogeneity, and the deviance information criterion. In a well-fitting model, the posterior mean residual deviance should be close to the number of data points [34, 35]. A difference of three units in the deviance information criterion was considered important and the lowest value of the deviance information criterion corresponded to the model with the best fit $[34,35]$.

The safety of AED medications was ranked using the surface under the cumulative ranking (SUCRA) curve [36]. The larger the SUCRA value for a treatment, the higher its safety rank among all the available treatment options. Ideally, we would like to observe a steep gradient in the SUCRA curve suggesting that the corresponding treatment is most likely the safest. SUCRA curves are presented along with 95\% CrIs. A rank-heat plot was used to depict the SUCRA values for all outcomes (http://rh.ktss.ca/) [37].

Meta-analyses and NMAs were performed within OpenBUGS [38], assuming non-informative priors for all model parameters and a half-normal prior distribution for the between-study standard deviation $(\tau \sim N(0,1), \tau>0)$. The models were run for 100,000 iterations to ensure model convergence, which was checked by visual inspection of the mixing of two chains, after discarding the first 10,000 iterations and thinning of 10 . These samples were used to calculate the median and 95\% credible intervals (CrI) for each parameter value. Medians were presented instead of means, since means may be overly influenced by outliers. The design-by-treatment interaction model was performed in Stata using the network command [39]. The meta-analysis and NMA ORs were presented with 95\% CrIs for each pair of treatments. For the NMA effect estimates, a 95\% predictive interval (PrI) was also presented, capturing the magnitude of $\tau^{2}$ and presenting the interval within which we would expect the treatment effect of a future study to lie $[40,41]$.
In the following sections, the terms 'safer' and 'harmful' are used to indicate when a treatment is associated with a lower risk (safer) or greater risk (harmful) of experiencing an adverse outcome compared to the alternative (e.g., another AED or control).

\section{Results \\ Literature search}

After screening 5305 titles and abstracts, 642 potentially relevant full-text articles, and 17 additional studies identified from scanning reference lists, 154 publications describing 110 different studies were included (Fig. 1). Of the included 110 studies, nine were written in languages other than English and three were conference abstracts or letters to the editor with usable data. Scanning of reference lists of included articles and related reviews identified 13 additional studies. Overall, 48\% (22/46) of contacted authors responded to our query but only $17 \%$ (8/46) were able to provide additional data for our analysis. Further, 29\% (13/45) of authors of conference abstracts responded to our query but none were able to provide unpublished data for our analysis. We were unable to contact 11 authors due to non-working email addresses. One author provided a manuscript and four authors provided unpublished data that were included in the analysis.

Seventeen of the eligible studies reported neurological outcomes that were excluded in this paper and reported in another paper (personal communication with Dr. Veroniki), leaving 96 studies with 58,461 patients (reported in 93 articles) included for analysis (Additional file 3: Appendix B). A table of key studies excluded due to reporting only one treatment arm with abstractable data is provided in Additional file 3: Appendix C.

\section{Study and patient characteristics}

We included 92 cohort studies, three case-control studies, and one RCT (Table 1, Additional file 3: Appendices D and E) published between 1964 and 2015. The number of patients included per study ranged from 18 to 7759 . The most common study indication was epilepsy (93\%), and almost half of the studies (49\%) included unmedicated women with epilepsy as a control group. The mean maternal age ranged from 24 to 34 years. Most studies (58\%) were conducted in Europe, followed by North America (19\%).

\section{Methodological quality/risk-of-bias}

The RCT was appraised with the Cochrane risk-of-bias tool and had an unclear risk-of-bias for reporting bias and 'other' bias (i.e., funding bias), as well as a high riskof-bias for random sequence generation and allocation concealment (Additional file 3: Appendix F). Three casecontrol studies and 92 cohort studies were assessed with 


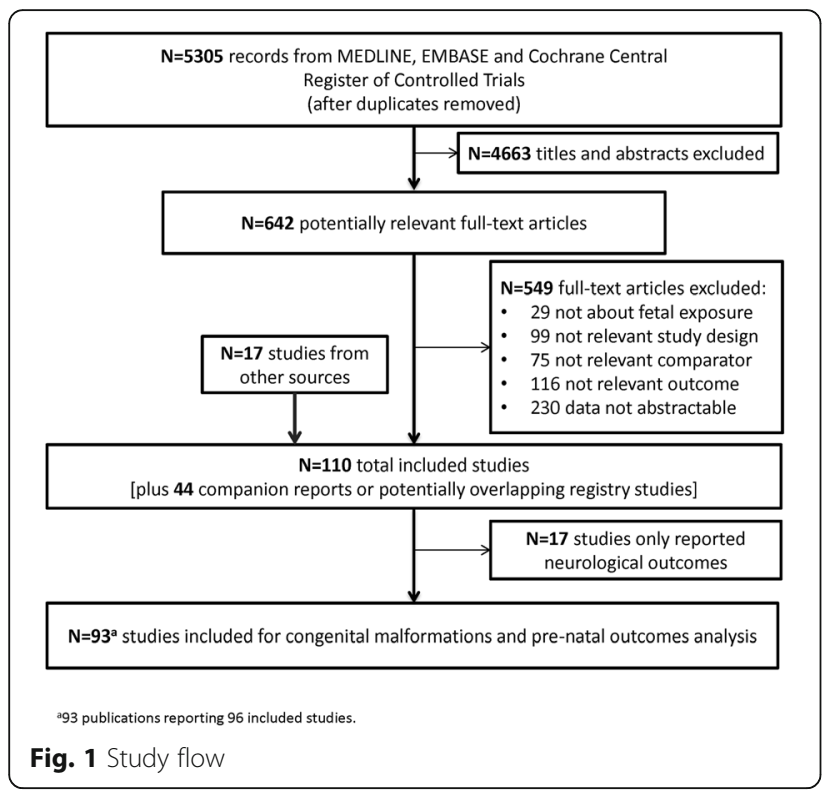

the Newcastle-Ottawa Scale. The case-control studies had high methodological quality on all items except for the comparability of cohorts on the basis of the design/ analysis (Additional file 3: Appendix G). Methodological shortcomings in the cohort studies (Additional file 3: Appendix $\mathrm{H}$ ) included not controlling for confounders (81\%) or reporting number of patients lost to follow-up (59\%). The comparison-adjusted funnel plots showed no evidence for publication bias and small-study effects across all outcomes (Additional file 3: Appendix I).

\section{Statistical analysis}

The transitivity assumption was upheld for mean age, mean baseline risk, treatment indication, and timing (Additional file 3: Appendix J). However, the adequacy of follow-up and comparability of cohort items varied across treatment comparisons. The design-by-treatment interaction model suggested that there was no evidence of statistically significant inconsistency for all outcomes and additional analyses (Additional file 3: Appendix J).

In the following sections, the overall NMA, metaregression, subgroup, and sensitivity analyses results for each outcome are discussed; the SUCRA curve results are presented in Fig. 2 and Additional file 3: Appendix K. Furthermore, AED sample sizes and absolute risks for each AED can be found in Additional file 3: Appendix K.

\section{Overall major $\mathrm{CMs}$}

The median baseline risk of major $\mathrm{CM}$ in the control group (no AED exposure) across all studies was 0.026 (interquartile range, 0.000-0.092; Additional file 3: Appendix K). The NMA on overall major CMs included 75 cohort studies, two case-control studies and one RCT, 35,016 cases, 47 AEDs plus control, with 15\% of all pairwise comparisons reaching statistical significance (Fig. 3a Additional file 3: Appendices J and L). The following monotherapies were associated with statistically significantly more cases developing major $\mathrm{CMs}$ than control: ethosuximide (OR, 3.04; 95\% CrI, 1.23-7.07), valproate (OR, 2.93; 95\% CrI, 2.36-3.69), topiramate (OR, 1.90; 95\% CrI, 1.17-2.97), phenobarbital (OR, 1.83; 95\% CrI, 1.35-2.47), phenytoin (OR, 1.67; 95\% CrI, 1.30-2.17), and carbamazepine (OR, 1.37; 95\% CrI, 1.10-1.71) (Fig. 4a). Gabapentin (OR, 1.00; 95\% CrI, 0.47-1.89), lamotrigine (OR, 0.96; 95\% CrI, 0.72-1.25), levetiracetam (OR, 0.72; 95\% CrI, 0.43-1.16), and nine polytherapies lacked sufficient evidence to reach statistical significance (Fig. 4a).

The results in subgroup NMA when restricting to observational studies only ( 2 case-control and 75 cohort studies, 34,966 cases, 48 treatments; $\tau^{2}=0.03$; 95\% CrI, $0.00-0.13)$ were in agreement with NMA. The sensitivity analysis restricting to cohort studies (75 studies, 34,667 cases, 48 treatments; $\tau^{2}=0.02 ; 95 \%$ CrI, $0.00-0.11$ ) found comparable results with NMA, but clonazepam plus valproate was marginally not statistically significant (OR, 12.780; 95\% CrI, 0.974-68.810). Similar results were also observed with the Schmitz model (1 RCT, 2 case-control, and 75 cohort studies, 35,016 cases, 48 treatments; $\tau^{2}=0.30 ; 95 \%$ CrI, $0.00-3.95$ ), but carbamazepine versus control was not statistically significant (OR, 1.34; 95\% CrI, 0.27-5.02) similar to the results obtained from the RCT ( 1 study, 50 cases, 3 treatments).

Similar results to the NMA were found with the sensitivity analysis including the EURAP study (1 RCT, 2 case-control, and 73 cohort studies, 48 treatments, 38,151 cases; $\tau^{2}=0.04 ; 95 \%$ CrI, 0.00-0.13), where control had statistically significantly lower risk of major $\mathrm{CM}$ than valproate combined with carbamazepine and phenytoin (OR, 6.14; 95\% CrI, 1.06-29.14) or with lamotrigine (OR, 2.94; 95\% CrI, 1.61-5.05), but did not have a significantly lower risk of major CM than ethosuximide (OR, 3.13; 95\% CrI, 0.77-6.59). The Schmitz model for the sensitivity analysis including the EURAP (1 RCT, 2 case-control, and 73 cohort studies, 38,151 cases, 48 treatments; $\tau^{2}=0.31$; 95\% CrI, 0.00-3.58) suggested a statistically significant OR for the comparison lamotrigine plus valproate versus control (OR, 3.01; 95\% CrI, 1.60-5.27), whereas clonazepam plus valproate $(\mathrm{OR}, 11.17 ; 95 \% \mathrm{CrI}, 0.77-66.36)$ and carbamazepine (OR, 1.32; 95\% CrI, 0.26-4.64) did not statistically significantly differ from control.

The sensitivity analysis results for timing of first trimester exposure to AED (1 RCT and 49 cohort studies, 25,329 cases, 46 treatments; $\tau^{2}=0.04 ; 95 \%$ CrI, $0.00-0.17$ ) for treatment indication of epilepsy (1 RCT, 2 case-control, and 68 cohort studies, 30,289 cases, 47 treatments; $\tau^{2}=0.03$; 95\% CrI, 0.00-0.13) and for older AEDs (i.e., without control, gabapentin, lamotrigine, levetiracetam, oxcarbazepine, 
Table 1 Summary characteristics of included studies

\begin{tabular}{|c|c|c|}
\hline Characteristic & $\begin{array}{l}\text { Number } \\
\text { of studies } \\
(n=96)\end{array}$ & $\begin{array}{l}\text { Percentage } \\
\text { of total }\end{array}$ \\
\hline \multicolumn{3}{|l|}{ Year of publication } \\
\hline 1964-1980 & 7 & 7.29 \\
\hline 1981-1990 & 19 & 19.79 \\
\hline $1991-2000$ & 22 & 22.92 \\
\hline $2001-2005$ & 8 & 8.33 \\
\hline 2006-2010 & 12 & 12.50 \\
\hline $2011-2015$ & 28 & 29.17 \\
\hline \multicolumn{3}{|l|}{ Continent } \\
\hline Europe & 56 & 58.33 \\
\hline North America & 18 & 18.75 \\
\hline Asia & 10 & 10.42 \\
\hline Trans-Continental & 5 & 5.21 \\
\hline Australia & 3 & 3.13 \\
\hline South America & 3 & 3.13 \\
\hline Africa & 1 & 1.04 \\
\hline \multicolumn{3}{|l|}{ Study design } \\
\hline Observational cohort & 92 & 95.83 \\
\hline Case-control & 3 & 3.13 \\
\hline Randomized clinical trial & 1 & 1.04 \\
\hline \multicolumn{3}{|l|}{ Registry study } \\
\hline Yes & 30 & 31.25 \\
\hline No & 66 & 68.75 \\
\hline \multicolumn{3}{|l|}{ Sample size } \\
\hline $18-50$ & 16 & 16.67 \\
\hline $51-100$ & 26 & 27.08 \\
\hline $101-300$ & 32 & 33.33 \\
\hline $301-500$ & 8 & 8.33 \\
\hline $501-1000$ & 2 & 2.08 \\
\hline $1001-7759$ & 12 & 12.50 \\
\hline \multicolumn{3}{|l|}{ Number of interventions ${ }^{a}$} \\
\hline $2-4$ & 41 & 42.71 \\
\hline $5-7$ & 30 & 31.25 \\
\hline $8-10$ & 15 & 15.63 \\
\hline $11-17$ & 10 & 10.42 \\
\hline \multicolumn{3}{|l|}{ Funding } \\
\hline Public & 21 & 21.88 \\
\hline Private & 7 & 7.29 \\
\hline Mixed public and private & 16 & 16.67 \\
\hline Not reported & 52 & 54.17 \\
\hline \multicolumn{3}{|l|}{ Indication } \\
\hline Epilepsy & 89 & 92.71 \\
\hline Mixed indications & 1 & 1.04 \\
\hline
\end{tabular}

Table 1 Summary characteristics of included studies (Continued)

\begin{tabular}{lll}
\hline Mental illness & 1 & 1.04 \\
Not reported & 5 & 5.21 \\
Epileptic control group & & \\
Yes & 47 & 48.96 \\
No/not reported/not applicable & 49 & 51.04 \\
Mean maternal age, years & & \\
24-26 & 11 & 11.46 \\
27-29 & 23 & 23.96 \\
30-34 & 7 & 7.29 \\
Not reported & 55 & 57.29 \\
Anti-epileptic drug exposure timing & & \\
At least 1st trimester & 64 & 66.67 \\
No/not reported & 32 & 33.33 \\
Folic acid use & & \\
Reported & 13 & 13.54 \\
Not reported & 83 & 86.46 \\
Alcohol use & & \\
Reported & 56.58 \\
Not reported & 5.21 \\
Tobacco use & 91 & 94.79 \\
Reported & & \\
Not reported & & \\
\hline n & & \\
\hline
\end{tabular}

ancluding any relevant control group

topiramate, and vigabatrin; 1 RCT, 2 case-control, and 50 cohort studies, 6982 cases, 31 treatments; $\tau^{2}=0.08$; 95\% CrI, 0.00-0.27) overall agreed with NMA. However, in timing, the polytherapy carbamazepine plus phenytoin plus valproate was associated with statistically significantly more cases developing major CMs than control (OR, 8.00; 95\% CrI, 1.02-32.61), whereas clonazepam plus valproate (OR, 13.34; 95\% CrI, 0.21-90.51) and ethosuximide (OR, 2.80; 95\% CrI, 0.93-6.52) did not statistically differ from control.

Five cohort studies of 5212 women with a history of alcohol comparing 16 treatments $\left(\tau^{2}=0.20 ; 95 \% \mathrm{CrI}\right.$, $0.00-1.49)$ and two cohort studies comparing 11 treatments in 5057 women reported a family history of CMs $\left(\tau^{2}=0.23\right.$; 95\% CrI, 0.00-3.42), suggesting that no AED was statistically significantly different than control. Another 5 cohort studies that reported folic acid use in more than $50 \%$ of the 10,825 included women compared 15 treatments and showed that valproate was statistically significantly more harmful than control (OR, 2.86; 95\% CrI, 1.18-6.22; $\tau^{2}=0.09 ; 95 \%$ CrI, 0.00-0.72).

To assess the impact of small studies, we conducted a NMA restricted to studies including more than 300 cases. We included 13 cohort studies, 27,227 cases, and 22 treatments $\left(\tau^{2}=0.03\right.$; 95\% CrI, $\left.0.00-0.17\right)$, and the 


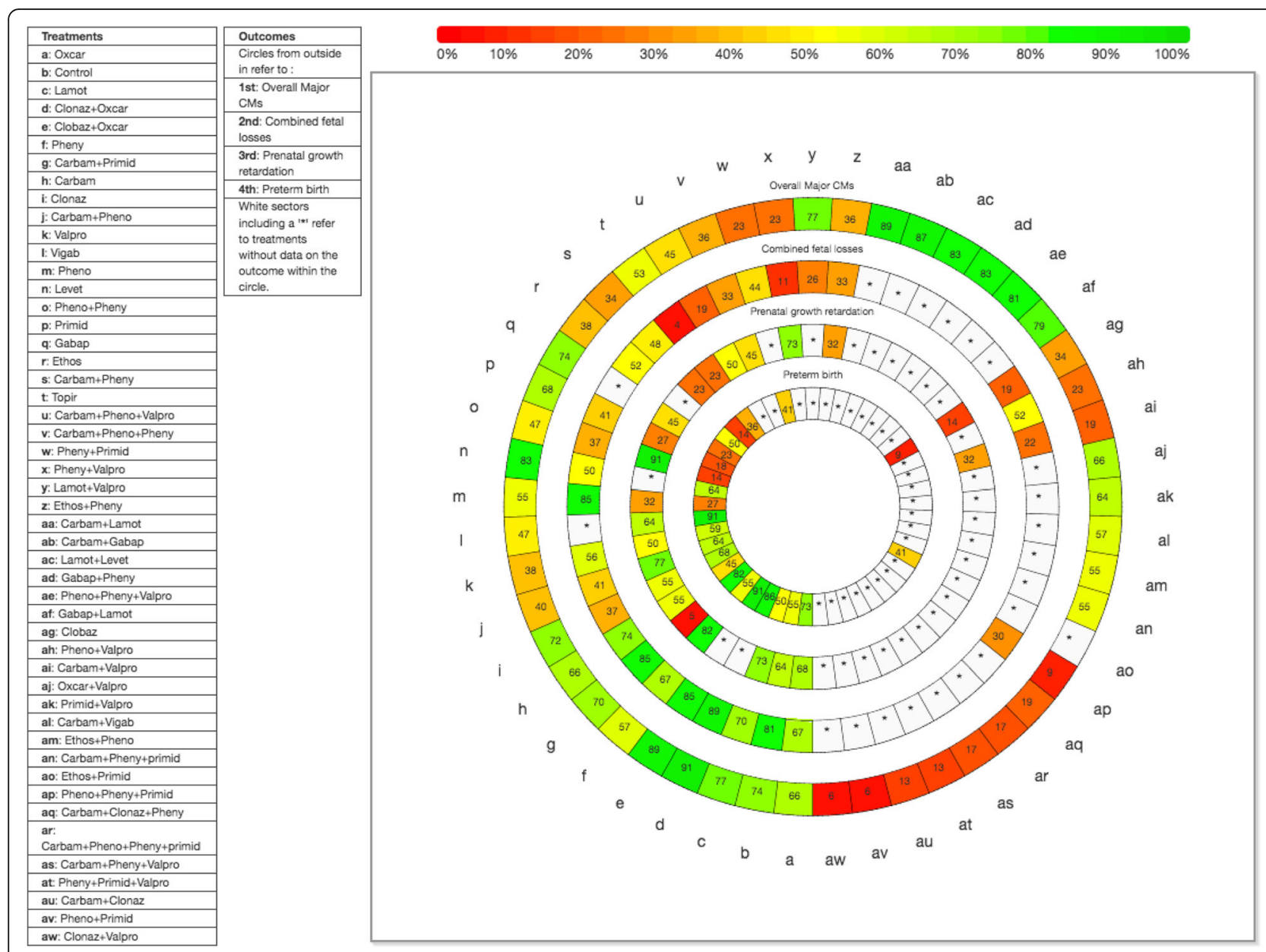

Fig. 2 Rank heat plot for overall major congenital malformations (CMs), combined fetal losses, prenatal growth retardation, and preterm birth. Rank-heat plot of 49 treatments (presented in 49 radii) and four outcomes (presented in four concentric circles). Each sector is colored according to the SUCRA value of the corresponding treatment and outcome using the transformation of three colors: red (0\%), yellow (50\%), and green (100\%). carbam carbamazepine, clobaz clobazam, clonaz clonazepam, ethos ethosuximide, gabap gabapentin, lamot lamotrigine, levet levetiracetam, oxcar oxcarbazepine, pheno phenobarbital, pheny phenytoin, primid primidone, topir topiramate, valpro valproate, vigab vigabatrin

sensitivity analysis suggested that carbamazepine plus phenytoin plus valproate was associated with statistically significantly more cases developing major CMs compared to control (OR, 20.77; 95\% CrI, 1.72-154.20), whereas clonazepam plus valproate (OR, 11.65; 95\% CrI, 0.82-71.86) did not statistically differ from the control. The sensitivity analysis for low risk-of-bias in the comparability of cohorts item on the Newcastle-Ottawa Scale, including 10 observational studies, 21,622 cases, and 31 treatments $\left(\tau^{2}=0.03\right.$; 95\% CrI, 0.00-0.21), suggested that only phenobarbital (OR, 2.22; 95\% CrI, 1.124.08), topiramate (OR, 1.89; 95\% CrI, 1.10-3.24), and valproate (OR, 2.77; 95\% CrI, 1.92-4.09) were statistically significantly different from the control. When restricting to low risk-of-bias for the adequacy of followup of cohorts $\left(\mathrm{k}=35, \mathrm{n}=20,122 ; \tau^{2}=0.05 ; 95 \% \mathrm{CrI}\right.$, $0.00-0.22$ ), phenytoin plus primidone (OR, 2.58; 95\% CrI, 0.46-9.77), phenytoin plus valproate (OR, 1.90; 95\%
CrI, 0.23-8.94), and topiramate (OR, 1.59; 95\% CrI, 0.63-3.40) were no longer statistically significantly different from zero.

Accounting for baseline risk in a network metaregression model resulted in a statistically non-significant association with the treatment effect ( 1 RCT, 2 casecontrol, and 75 cohort studies, 35,016 cases, 48 treatments, estimated regression coefficient on OR scale, 1.02; 95\% CrI, 0.93-1.10; $\tau^{2}=0.03 ;$ 95\% CrI, 0.000.14 ; residual deviance $=411$, data points $=468$, deviance information criterion $=562$ ). Similarly, a statistically significant association was not observed in our network meta-regression analysis conducted using age as a covariate (32 cohort studies, 15,948 cases, 43 treatments, estimated regression coefficient on OR scale, 0.99; 95\% CrI, 0.85-1.15; $\tau^{2}=0.03 ; 95 \%$ CrI, $0.00-0.16$; residual deviance $=180$, data points $=213$, deviance information criterion $=267$ ). For more details 


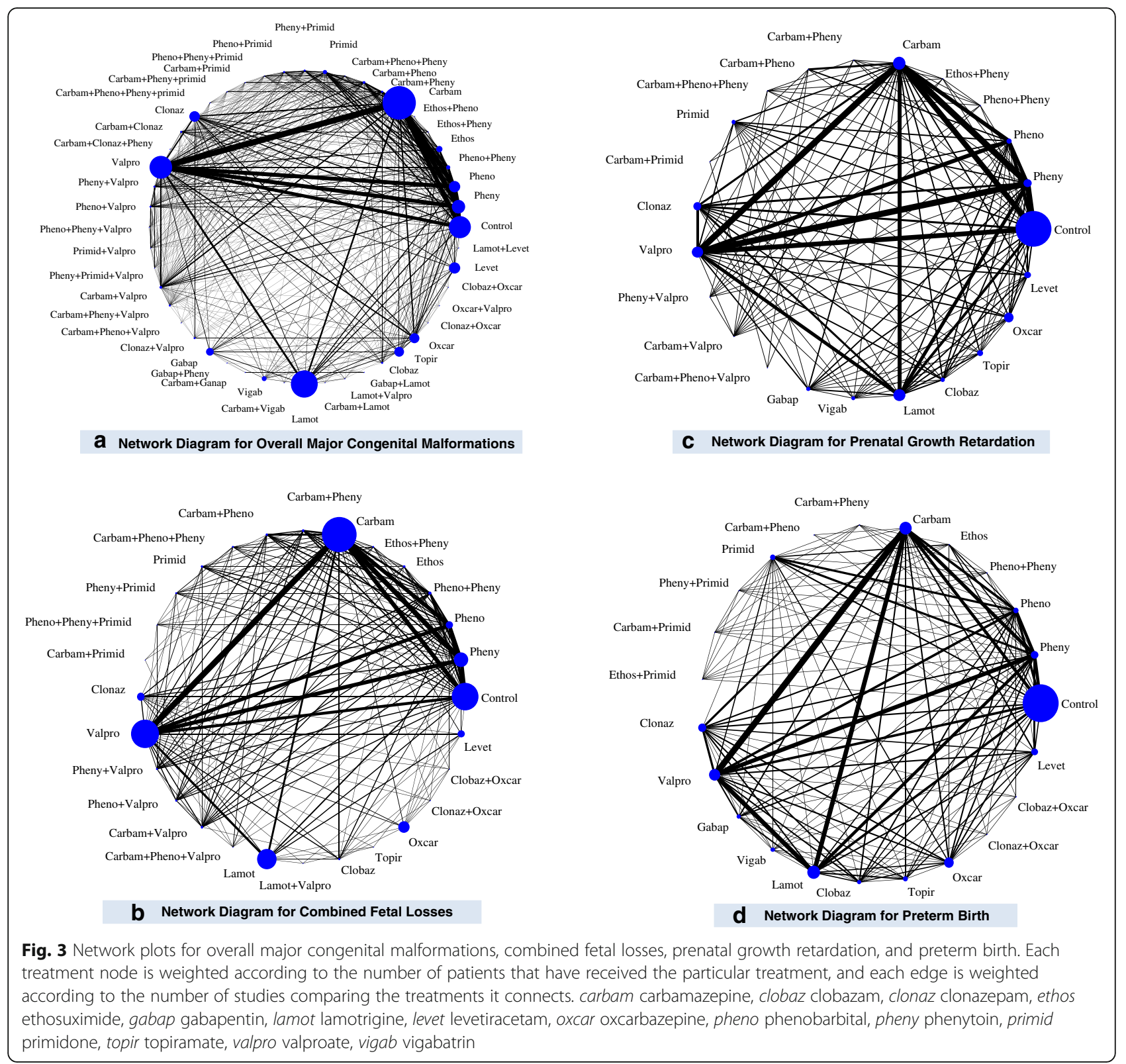

on the subgroup, meta-regression, and sensitivity analyses see Additional file 3: Appendix M).

\section{Combined fetal losses}

The median baseline risk of combined fetal losses in the control group (no AED exposure) across all studies was 0.000 (interquartile range: $0.000-0.000$; Additional file 3 : Appendix K). The NMA for combined fetal losses included 1 RCT, 1 case-control study, and 29 cohort studies, 13,487 pregnancies, and 27 AEDs plus control, with $5 \%$ of comparisons reaching statistical significance (Fig. 3b; Additional file 3: Appendices A, J and L). Topiramate (OR, 23.58; 95\% CrI, 1.18-549.60), primidone (OR, 2.81; 95\% CrI, 1.21-6.28), valproate (OR, 1.83; 95\%
CrI, 1.04-3.45), and two polytherapies (carbamazepine plus valproate: OR, 5.09; 95\% CrI, 1.35-16.79; phenytoin plus valproate: OR, 8.96; 95\% CrI, 1.77-37.95) were associated with statistically significantly more combined fetal losses than control (Fig. 4b).

Similar results with the NMA analyses were observed in subgroup analysis including observational studies only (1 case-control and 29 cohort studies, 13,437 pregnancies; $\tau^{2}=0.03$; 95\% CrI, 0.00-0.26) and in the Schmitz model (1 RCT, 1 case-control study, and 29 cohort studies, 13,487 pregnancies; $\tau^{2}=0.36$; 95\% CrI, 0.00-4.17), where control was additionally associated with a marginally statistically significantly lower risk of fetal loses than the combination phenobarbital and phenytoin (OR, 3.04; 


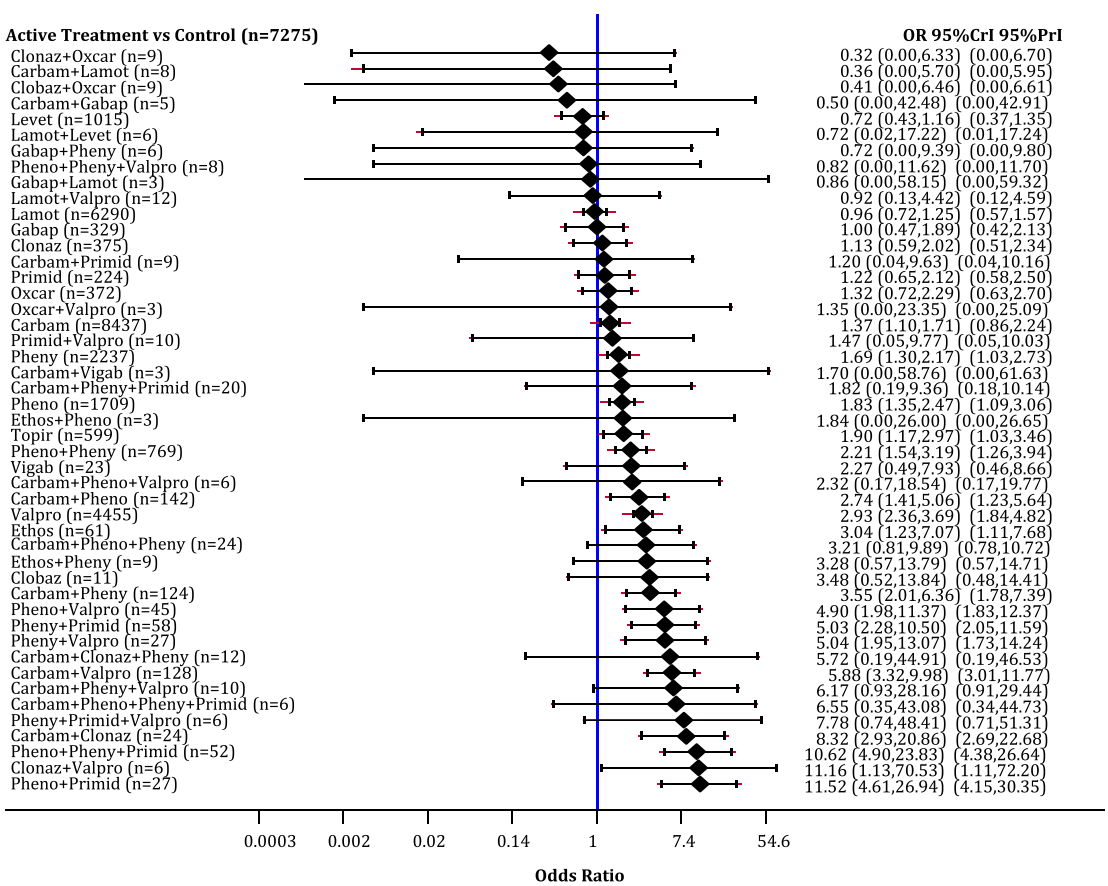

\section{b}

Combined Fetal Losses

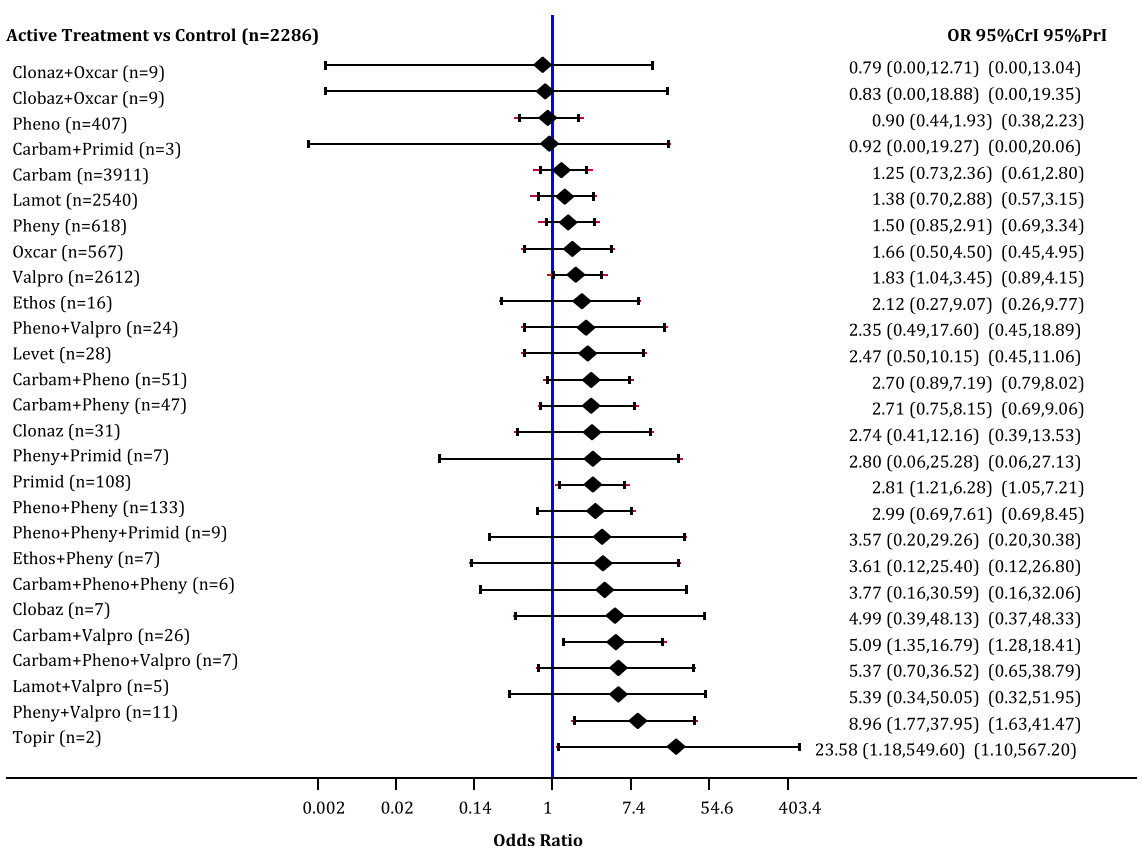

Fig. 4 Network meta-analysis forest plots for each treatment versus control. Each rhombus represents the summary treatment effect estimated in the network meta-analysis on the odds ratio (OR) scale. The black horizontal lines represent the credible intervals (Crl) for the summary treatment effects, and the red horizontal lines represent the corresponding predictive intervals (Prl). In the absence of heterogeneity, the Crls and Prls should be identical. An OR $>1$ suggests that control is safer, whereas an $O R<1$ suggests that the comparator active treatment is safer. The vertical blue line corresponds to an $\mathrm{OR}=1$ (i.e., the treatment groups compared are equally safe). The total sample size (n) included in each treatment is also presented. a Overall major congenital malformations (78 studies, 35,016 cases, 48 treatments). b Combined fetal losses (31 studies, 13,487 cases, 28 treatments). carbam carbamazepine, clobaz clobazam, clonaz clonazepam, ethos ethosuximide, gabap gabapentin, lamot lamotrigine, levet levetiracetam, oxcar oxcarbazepine, pheno phenobarbital, pheny phenytoin, primid primidone, topir topiramate, valpro valproate, vigab vigabatrin 
95\% CrI, 1.07-7.18), except for topiramate (OR, 13.06; 95\% CrI, 0.77-365.50). The sensitivity analysis results for timing of at least first trimester exposure to AED (1 case-control and 16 cohort studies, 6970 pregnancies; $\tau^{2}=0.04 ; 95 \% \mathrm{CrI}, 0.00-0.17$ ) were in agreement with NMA, and the only statistically significant results of all treatments versus control were for carbamazepine combined with valproate (OR, 7.83; 95\% CrI, 1.62-32.08) or phenobarbital (OR, 4.73; 95\% CrI, 1.24-17.24), with control statistically significantly safer. Two cohort studies with 318 women with a history of alcohol use during pregnancy compared 10 treatments $\left(\tau^{2}=0.31 ; 95 \%\right.$ CrI, 0.00-3.87) and another 3 cohort studies with 4666 women with a smoking history compared 14 treatments $\left(\tau^{2}=0.14 ; 95 \%\right.$ CrI, 0.00-2.19), and showed that only phenytoin plus valproate was statistically significantly different than control (alcohol use: OR, 269.30; 95\% CrI, 2.42-1.19 × 106 , smoking history: OR, 180.30; 95\% CrI, $\left.6.10-4.17 \times 10^{5}\right)$. The restriction to studies comparing only older AEDs (1 RCT, 1 casecontrol, and 20 cohort studies, 3054 neonates; $\tau^{2}=0.06$; $95 \%$ CrI, 0.00-0.49) suggested that control was associated with a marginally statistically significantly lower risk of fetal loses than phenobarbital plus phenytoin (OR, 2.93; 95\% CrI, 1.04-7.73), whereas valproate (OR, 1.76; 95\% CrI, 0.86-3.82) was no longer statistically significantly different than control.

The sensitivity analyses restricting to (1) studies with more than 300 pregnancies (4 cohort studies, 10,224 women, 10 treatments; $\tau^{2}=0.25 ; 95 \%$ CrI, 0.00-2.05), (2) low risk-of-bias in the "comparability of cohorts" item on the Newcastle-Ottawa Scale (2 cohort studies, 5539 women, 4 treatments; $\tau^{2}=0.75$; 95\% CrI, 0.00-5.42), and (3) low risk-of-bias for the "adequacy of follow-up of cohorts" item (15 cohort studies, 6236 women, 23 treatments; $\tau^{2}=0.07 ; 95 \%$ CrI, 0.00-0.61) suggested no AED differed statistically significantly from the control. The network meta-regression analyses using baseline risk (1 RCT, 1 case-control study, and 29 cohort studies, 13,487 pregnancies, 28 treatments, estimated regression coefficient on OR scale, 1.00; 95\% CrI, 0.94-1.08; $\tau^{2}=0.05$; 95\% CrI, 0.00-0.31; residual deviance $=130$, data points $=$ 175 , deviance information criterion $=199)$ and age $(1$ case-control study, 14 cohort studies, 7152 pregnancies, 22 treatments, estimated regression coefficient on OR scale, 0.92; 95\% CrI, 0.67-1.33; $\tau^{2}=0.09 ; 95 \%$ CrI, 0.000.58 ; residual deviance $=74$, data points $=96$, deviance information criterion $=118$ ) as covariates suggested no statistically significant associations with the treatment effect (Additional file 3: Appendix M).

\section{Prenatal growth retardation}

The median baseline risk of prenatal growth retardation in the control group (no AED exposure) across all studies was 0.047 (interquartile range, 0.024-0.100; Additional file 3: Appendix K). The NMA for prenatal growth retardation included 16 cohort studies, 18,117 children, 22 AEDs plus control, with $8 \%$ of comparisons reaching statistical significance (Fig. 3c; Additional file 3: Appendices A, J and L). Clobazam (OR, 4.47; 95\% CrI, 1.60-11.18), topiramate (OR, 2.64; 95\% CrI, 1.41-4.63), and phenobarbital (OR, 1.88; 95\% CrI, 1.07-3.32) were associated with statistically significantly more children experiencing prenatal growth retardation than control (Fig. 5a).

The sensitivity analysis results for timing of at least first trimester exposure to AED (6 cohorts, 16,263 children, 14 treatments; $\left.\tau^{2}=0.09 ; 95 \% \mathrm{CrI}, 0.00-0.55\right)$ and for treatment indication of epilepsy (15 cohorts, 18,099 children, 23 treatments; $\tau^{2}=0.10 ; 95 \%$ CrI, 0.00-0.37) were in agreement with the NMA, where control was not significantly safer than phenobarbital (timing: OR, 1.85; 95\% CrI, 0.92-3.97; epilepsy: OR, 1.79; 95\% CrI, 1.00-3.10). However, control was associated with a statistically significant lower risk of prenatal growth than carbamazepine for first trimester exposure (OR, 1.51; 95\% CrI, 1.01-2.46). The subgroup NMA for different AED generations showed that no AED was statistically significantly different from control, whereas the safest agent when comparing the newer AEDs (topiramate and lamotrigine) was lamotrigine (1 cohort study, 1928 children, 2 treatments; OR, 3.03; 95\% CrI, 2.13-4.17). One cohort study with 308 women with a history of alcohol use showed that lamotrigine was statistically significantly better than carbamazepine (OR, 0.29; 95\% CrI, 0.090.93) and valproate (OR, 0.25; 95\% CrI, 0.07-0.85), but not significantly safer than phenytoin (OR, 0.89; 95\% CrI, 0.16-5.00). Six cohort studies with 16,263 women with a smoking history compared 14 treatments $\left(\tau^{2}=\right.$ 0.09 ; 95\% CrI, 0.00-0.55) and suggested that only clobazam (OR, 4.07; 95\% CrI, 1.24-11.61) and topiramate (OR, 2.79; 95\% CrI, 1.43-5.25) were associated with statistically significantly more children experiencing prenatal growth retardation than control.

The restriction to large studies (>300 patients) included 7 cohort studies, 16,899 children, and 14 treatments $\left(\tau^{2}=\right.$ 0.12; 95\% CrI, 0.01-0.51) suggesting that only clobazam (OR, 3.73; 95\% CrI, 1.11-11.26) was associated with statistically significantly more children experiencing prenatal growth retardation than control. The sensitivity analysis for low risk-of-bias in the "comparability of cohorts" item, including 7 cohort studies, 16,502 children, and 15 treatments $\left(\tau^{2}=0.12\right.$; 95\% CrI $\left.0.00-0.57\right)$, suggested that no AED differed statistically significantly from control. When restricting to low risk-of-bias for the "adequacy of followup of cohorts" item (11 cohort studies, 15,200 children, 23 treatments; $\tau^{2}=0.10$; 95\% CrI, 0.00-0.46) clobazam (OR, 4.09; 95\% CrI, 1.26-11.82) and topiramate (OR, 2.88; 95\% CrI, 1.34-5.88) were associated with statistically significantly more children experiencing prenatal growth retardation than control. 
a

Active Treatment vs Control ( $n=7347)$

Pheny ( $\mathrm{n}=519)$

Carbam+Pheno (n=19)

Pheny+Valpro $(n=5)$

Lamot ( $\mathrm{n}=2882)$

Oxcar (n=1002)

Vigap $(\mathrm{n}=13)$

Clonaz ( $\mathrm{n}=411$ )

Carbam (n=2897)

Carbam+Pheno+Valpro ( $\mathrm{n}=3$ )

Levet $(\mathrm{n}=81)$

Valpro $(n=1622)$

Gabap (n=70)

Carbam+Pheno+Pheny ( $\mathrm{n}=4)$

Pheno $(\mathrm{n}=400)$

Ethos+Pheny ( $\mathrm{n}=3)$

Primid $(\mathrm{n}=52)$

Topir ( $n=472)$

Carbam+Pheny ( $\mathrm{n}=11)$

Clobaz $(n=34)$

Carbam+Primid $(\mathrm{n}=3)$
Pheno+Pheny ( $\mathrm{n}=15)$

Carbam+Valpro $(n=4)$

\section{Prenatal Growth Retardation}

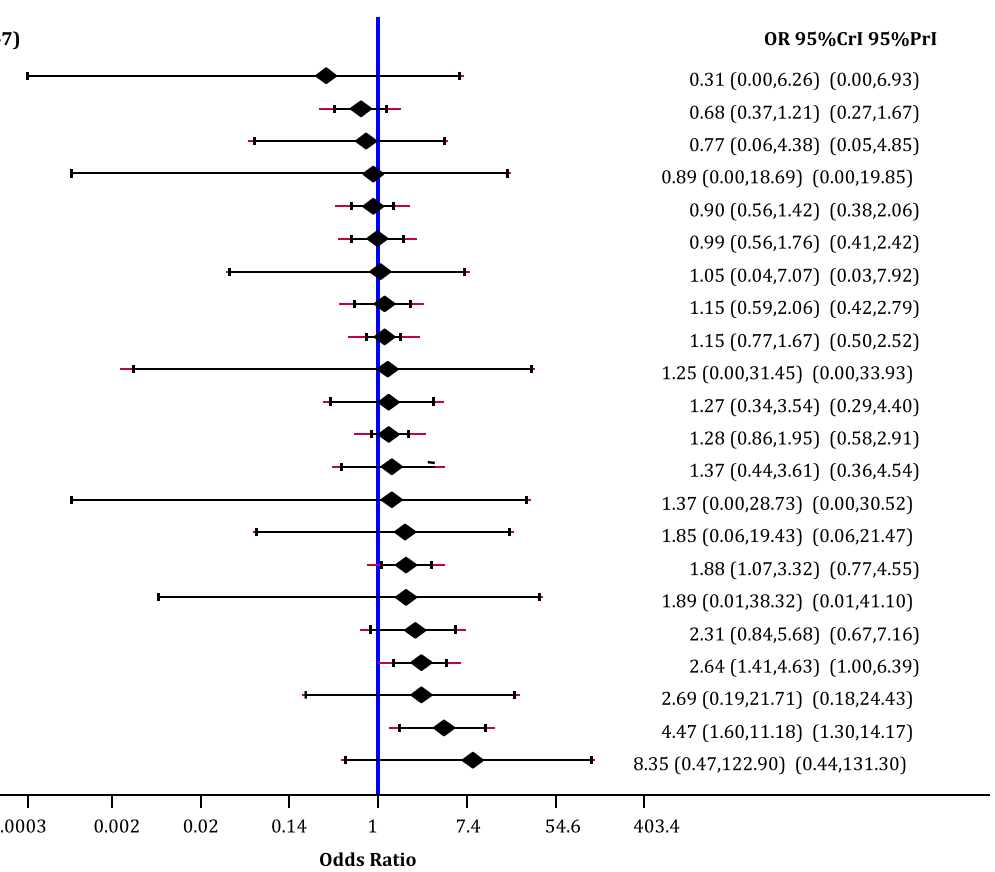

b

Preterm Birth

Active Treatment vs Control $(n=7404)$

Vigap ( $\mathrm{n}=13)$

Clonaz+0xcar (n=9)

Clobaz+Oxcar ( $\mathrm{n}=9$ )

Carbam+Primid ( $\mathrm{n}=10)$

Oxcar ( $n=1045)$

Clonaz ( $\mathrm{n}=437)$

Levet $(\mathrm{n}=93)$

Carbam+Pheno (n=12)

Valpro ( $\mathrm{n}=1694)$

Pheny ( $\mathrm{n}=283)$

Lamot $(n=3015)$

Ethos ( $\mathrm{n}=19)$

Carbam (n=2141)

Ethos+Primid ( $\mathrm{n}=26)$

Pheny+Primid ( $\mathrm{n}=25)$

Topir $(n=408)$

Pheno $(\mathrm{n}=206)$

Gabap ( $n=76)$

Primid ( $n=168)$

Pheno+Pheny $(n=2)$

Carbam+Pheny $(\mathrm{n}=3)$

Clobaz ( $n=35)$
OR 95\%CrI 95\%PrI

$0.23(0.00,3.11)(0.00,3.38)$

$0.29(0.00,4.15)(0.00,4.37)$

$0.29(0.00,4.06)(0.00,4.24)$

$0.52(0.00,8.61)(0.00,8.99)$

$0.80(0.51,1.26)(0.41,1.57)$

$0.86(0.48,1.39)(0.40,1.69)$

$0.87(0.31,2.04)(0.28,2.25)$

$0.87(0.04,8.14)(0.04,8.58)$

$0.96(0.65,1.37)(0.51,1.75)$

$1.03(0.55,1.82)(0.46,2.15)$

$1.05(0.70,1.48)(0.54,1.89)$

$1.07(0.04,6.87)(0.04,7.24)$

$1.10(0.77,1.56)(0.60,2.03)$

$1.18(0.07,6.12)(0.07,6.52)$

$1.23(0.09,7.26)(0.08,7.52)$

$1.38(0.73,2.35)(0.61,2.75)$

$1.59(0.87,2.75)(0.74,3.27)$

$1.93(0.88,4.05)(0.77,4.66)$

$2.12(1.01,4.27)(0.89,4.94)$

$2.40(0.00,57.25)(0.00,58.98)$

$2.56(0.01,41.12)(0.01,41.42)$

$3.42(1.41,7.92)(1.26,9.14)$

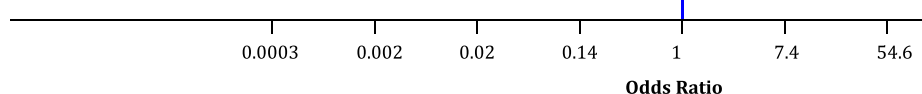

Fig. 5 Network meta-analysis forest plots for each treatment versus control. Each rhombus represents the summary treatment effect estimated in the network meta-analysis on the odds ratio (OR) scale. The black horizontal lines represent the credible intervals (Crl) for the summary treatment effects, and the red horizontal lines represent the corresponding predictive intervals (Prl). In the absence of heterogeneity, the Crls and Prls should be identical. An OR > 1 suggests that control is safer, whereas an OR $<1$ suggests that the comparator active treatment is safer. The vertical blue line corresponds to an $\mathrm{OR}=1$ (i.e., the treatment groups compared are equally safe). The total sample size ( $\mathrm{n}$ ) included in each treatment is also presented. a Prenatal growth retardation (16 studies, 18,177 cases, 23 treatments). b Preterm birth (17 studies, 17,133 cases, 23 treatments). carbam carbamazepine, clobaz clobazam, clonaz clonazepam, ethos ethosuximide, gabap gabapentin, lamot lamotrigine, levet levetiracetam, oxcar oxcarbazepine, pheno phenobarbital, pheny phenytoin, primid primidone, topir topiramate, valpro valproate, vigab vigabatrin 
A network meta-regression analysis using baseline risk as a covariate was conducted and a statistically significant association with the treatment effect was not detected, despite a slight drop in the between-study variance (16 cohort studies, 18,117 children, 23 treatments, estimated regression coefficient on OR scale, 0.82 ; $95 \%$ CrI, 0.67-1.00; $\tau^{2}=0.05 ; 95 \%$ CrI, 0.00-0.30; residual deviance $=87$, data points $=89$, deviance information criterion = 135, Additional file 3: Appendix M).

\section{Preterm birth}

The median baseline risk of preterm birth in the control group (no AED exposure) across all studies was 0.051 (interquartile range, 0.025-0.072; Additional file 3: Appendix K). The NMA on preterm birth included 17 cohort studies, 17,133 neonates, and 22 AEDs plus control, with $5 \%$ of comparisons reaching statistical significance (Fig. 3d, Additional file 3: Appendices A, J and L). Clobazam (OR, 3.42; 95\% CrI, 1.41-7.92) and primidone (OR, 2.12; 95\% CrI, 1.01-4.27) were associated with statistically significantly more preterm births than control (Fig. 5b).

\section{Cardiac malformations}

The median baseline risk of cardiac malformations in the control group (no AED exposure) across all studies was 0.000 (interquartile range, 0.000-0.027; Additional file 3: Appendix K). The NMA on cardiac malformations included 1 RCT, 1 case-control, and 49 cohort studies, 21,935 cases, 39 AEDs plus control, with $11 \%$ of comparisons reaching statistical significance (Additional file 3: Appendices J, L, and N). Levetiracetam (OR, 0.25; 95\% CrI, $0.03-0.96)$ and lamotrigine (OR, 0.55; 95\% CrI, 0.32-0.95) were monotherapies statistically significantly less likely to be associated with cases experiencing cardiac malformations than control. In contrast, gabapentin (OR, 5.98; 95\% CrI, 1.37-19.73), carbamazepine plus phenytoin (OR, 6.58; 95\% CrI, 2.25-18.97), phenobarbital plus valproate (OR, 8.01; 95\% CrI, 1.17-35.40), phenytoin plus valproate (OR, 8.88; 95\% CrI, 2.62-30.65), and carbamazepine plus clonazepam (OR, 10.08; 95\% CrI, 1.40-51.22) were associated with statistically significantly more cases developing cardiac malformations compared to control (Fig. 6a).

\section{Hypospadias}

The median baseline risk of hypospadias in the control group (no AED exposure) across all studies was 0.000 (interquartile range, 0.000-0.015; Additional file 3: Appendix K). The NMA for hypospadias included 1 RCT, 1 case-control, and 29 cohort studies, 12,365 cases, and 31 AEDs plus control, with $7 \%$ of comparisons reaching statistical significance (Additional file 3: Appendices J, L, and N). Gabapentin (OR, 16.54; 95\% CrI, 2.50-121.70), clonazepam (OR, 6.17; 95\% CrI, 1.17-24.80), primidone (OR, 5.92; 95\% CrI, 1.01-23.77), and valproate (OR, 2.58; $95 \%$
CrI, 1.24-5.76) were associated with statistically significantly more cases developing hypospadias compared to control (Fig. 6b).

\section{Cleft lip/palate}

The median baseline risk of cleft lip/palate in the control group (no AED exposure) across all studies was 0.000 (interquartile range, 0.000-0.000; Additional file 3: Appendix K). The NMA on cleft lip/palate included 1 RCT, 1 case-control, and 27 cohort studies, 18,987 cases, and 32 AEDs plus control, with $11 \%$ of comparisons reaching statistical significance (Additional file 3: Appendices J, L, and N). The following monotherapies were associated with statistically significantly more cases developing cleft lip/palate than control (Fig. 7a): ethosuximide (OR, 22.22; 95\% CrI, 4.56-87.64), primidone (OR, 7.68; 95\% CrI, 1.41-29.27), topiramate (OR, 6.12; 95\% CrI, 1.89-19.05), phenobarbital (OR, 5.75; 95\% CrI, 2.41-14.08), phenytoin (OR, 3.11; 95\% CrI, 1.317.72), and valproate (OR, 3.26; 95\% CrI, 1.38-5.58). In addition, the following polytherapies were associated with statistically significantly more cases developing cleft lip/ palate than control: phenobarbital plus phenytoin plus primidone (OR, 11.50; 95\% CrI, 1.70-63.48), phenytoin plus primidone (OR, 16.75; 95\% CrI, 3.02-77.19), carbamazepine plus phenobarbital (OR, 18.51; 95\% CrI, 3.34-94.21), and carbamazepine plus valproate (OR, 19.12; 95\% CrI, 3.74-88.68).

\section{Club foot}

The median baseline risk of club foot in the control group (no AED exposure) across all studies was 0.000 (interquartile range, 0.000-0.000; Additional file 3: Appendix K). The NMA for club foot included 1 RCT, 1 case-control, and 21 cohort studies, 8836 cases, and 26 AEDs plus control, with $7 \%$ of comparisons reaching statistical significance (Additional file 3: Appendices J, L, and N). Phenytoin (OR, 2.73; 95\% CrI, 1.13-6.18), valproate (OR, 3.26; 95\% CrI, 1.43-8.25), primidone (OR, 4.71; 95\% CrI, 1.11-17.24), ethosuximide (OR, 12.99; 95\% CrI, 1.6676.39), carbamazepine plus phenobarbital (OR, 7.30; 95\% CrI, 1.29-32.31), and phenobarbital plus phenytoin plus primidone (OR, 13.46; 95\% CrI, 1.45-132.80) were associated with statistically significantly more cases developing club foot than control (Fig. 7b).

\section{Inguinal hernia}

The median baseline risk of inguinal hernia in the control group (no AED exposure) across all studies was 0.000 (interquartile range, 0.000-0.000; Additional file 3: Appendix K). The NMA for inguinal hernia included 1 RCT, 1 case-control, and 11 cohort studies, 12,216 cases, and 28 AEDs plus control, with $8 \%$ of comparisons reaching statistical significance (Additional file 3: 
a

Cardiac Malformations

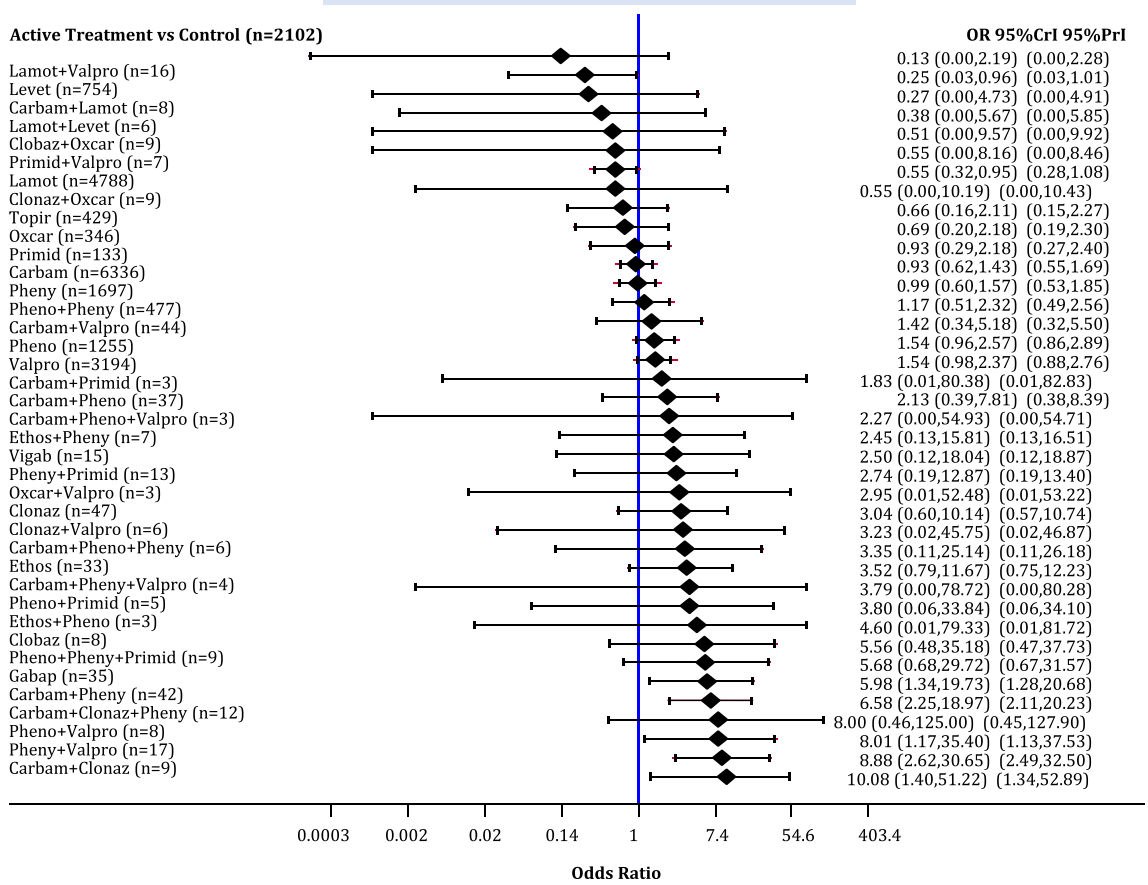

b

Hypospadias

Active Treatment vs Control $(\mathrm{n}=488)$ Levet ( $\mathrm{n}=754)$

Pheno+Pheny+Primid $(\mathrm{n}=9)$

Lamot+Valpro ( $\mathrm{n}=16)$

Lamot ( $\mathrm{n}=95)$

Pheno+Pheny ( $\mathrm{n}=175$ )

Carbam $(\mathrm{n}=3540)$

Pheny $(\mathrm{n}=1350)$

Carbam+Lamot $(\mathrm{n}=8)$

Pheno ( $n=1024)$

Lamot+Levet $(\mathrm{n}=6)$

Pheny+Valpro $(\mathrm{n}=9)$

Pheno+Primid ( $\mathrm{n}=2)$

Ethos+Pheny ( $\mathrm{n}=7)$

Pheny+Primid ( $\mathrm{n}=7$ )

Valpro $(\mathrm{n}=1437)$

Carbam+Pheny ( $\mathrm{n}=19$ )

Carbam+Pheno+Pheny ( $\mathrm{n}=6$ )

Topir $(n=429)$

Carbam+Valpro (n=34)

Carbam+Pheno $(\mathrm{n}=29)$

Clonaz+Valpro $(\mathrm{n}=6)$

Oxcar $(\mathrm{n}=200)$

Pheno+Valpro $(\mathrm{n}=6)$

Primid $(\mathrm{n}=74)$

Clonaz $(\mathrm{n}=42)$

Carbam+Clonaz $(\mathrm{n}=13)$

Carbam+Pheny+Valpro ( $\mathrm{n}=4$

Ethos ( $\mathrm{n}=31)$

Vigab $(\mathrm{n}=6)$

Clobaz ( $\mathrm{n}=6)$

Gabap (n=33)
OR 95\% CrI 95\% $\mathrm{Prl}$

$0.29(0.00,2.56)(0.00,2.78)$ $0.56(0.00,10.53)(0.00,10.96)$ $0.62(0.00,12.67)(0.00,13.02)$ $0.66(0.23,2.26)(0.19,2.60)$ $0.99(0.20,3.81)(0.18,4.25)$ $1.09(0.53,2.61)(0.43,3.17)$ $1.12(0.51,2.66)(0.40,3.23)$ $1.21(0.00,21.59)(0.00,23.01)$ $1.53(0.60,3.84)(0.52,4.59)$ $1.56(0.00,55.52)(0.00,56.61)$ $1.73(0.07,16.48)(0.07,18.41)$ $2.10(0.02,73.00)(0.02,78.16)$ $2.10(0.13,22.22)(0.11,24.19)$ $2.31(0.10,19.55)(0.09,21.27)$ $2.31(0.10,19.55)(0.09,21.27)$ $2.58(1.24,5.76)(0.99,7.06)$ $2.61(0.23,16.42)(0.21,17.71)$
$2.69(0.09,27.70)(0.08,29.78)$ $2.69(0.09,27.70)(0.08,29.78)$
$3.52(0.77,15.72)(0.69,17.68)$ $4.42(0.86,17.41)(0.74,19.65)$ $4.87(0.94,19.43)(0.85,22.48)$ $5.08(0.03,78.70)(0.03,87.07)$ $5.19(0.95,20.58)(0.86,23.98)$ $5.78(0.20,65.78)(0.20,70.26)$ $5.92(1.00,23.77)(0.93,27.21)$ $5.92(1.00,23.77)(0.93,27.21)$ $6.17(1.17,24.80)(1.04,27.47)$ $6.28(0.78,30.59)(0.67,34.12)$ $6.55(0.01,150.10)(0.01,161.70)$ $6.99(0.96,28.02)(0.91,32.42)$ $10.49(0.00,289.30)(0.00,309.90)$ $10.94(0.46,101.30)(0.45,107.70)$ $16.54(2.50,121.70)(2.27,135.20)$

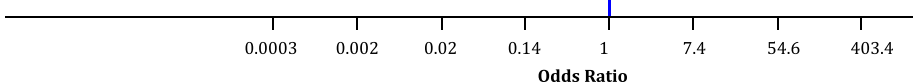

Fig. 6 Network meta-analysis forest plots for each treatment versus control. Each rhombus represents the summary treatment effect estimated in the network meta-analysis on the odds ratio (OR) scale. The black horizontal lines represent the credible intervals (Crl) for the summary treatment effects, and the red horizontal lines represent the corresponding predictive intervals (Prl). In the absence of heterogeneity, the Crls and Prls should be identical. An OR $>1$ suggests that control is safer, whereas an OR $<1$ suggests that the comparator active treatment is safer. The vertical blue line corresponds to an $\mathrm{OR}=1$ (i.e., the treatment groups compared are equally safe). The total sample size (n) included in each treatment is also presented. a Cardiac malformations (51 studies, 21,935 cases, 40 treatments). b Hypospadias (31 studies, 12,365 cases, 32 treatments). carbam carbamazepine, clobaz clobazam, clonaz clonazepam, ethos ethosuximide, gabap gabapentin, lamot lamotrigine, levet levetiracetam, oxcar oxcarbazepine, pheno phenobarbital, pheny phenytoin, primid primidone, topir topiramate, valpro valproate, vigab vigabatrin 
a

Active Treatment vs Control ( $n=1649$ )

Levet $(\mathrm{n}=872)$

Lamot (n=4664)

Carbam ( $n=5577)$

Pheno+Pheny (n=394)

Pheny ( $\mathrm{n}=1172)$

Valpro $(\mathrm{n}=2721)$

Oxcar $(\mathrm{n}=304)$

Carbam+Primid $(\mathrm{n}=3)$

Gabap $(\mathrm{n}=33)$

Pheny+Valpro $(\mathrm{n}=4)$

Carbam+Pheno+Valpro $(\mathrm{n}=6)$

Carbam+Clonaz $(\mathrm{n}=7)$

Pheno ( $\mathrm{n}=894)$

Topir $(\mathrm{n}=429)$

Pheno+Valpro $(\mathrm{n}=19)$

Primid $(\mathrm{n}=41)$

Carbam+Pheno+Pheny $(n=2)$

Ethos+Pheny $(\mathrm{n}=6)$

Pheno+Pheny+Primid ( $\mathrm{n}=27)$

Vigab $(\mathrm{n}=15)$

Clonaz+Valpro $(n=6)$

Conaz+Valpro $(\mathrm{n}=6)$

Clonaz ( $\mathrm{n}=18)$

Ethos + Pheno $(\mathrm{n}=3)$
Ox)

Ethos+Pheno $(\mathrm{n}=3)$

Oxcar+Valpro $(\mathrm{n}=3)$

Pheny+Primid $(\mathrm{n}=22)$

Carbam+Pheny+Valpro ( $\mathrm{n}=4)$

Carbam+Pheny+Valpro
Carbam+Pheno $(\mathrm{n}=16)$

Carbam+Pheno $(\mathrm{n}=16)$
Carbam+Valpro $(\mathrm{n}=26)$

Ethos $(\mathrm{n}=29)$

Clobaz (n=6)
Cleft Lip/Palate
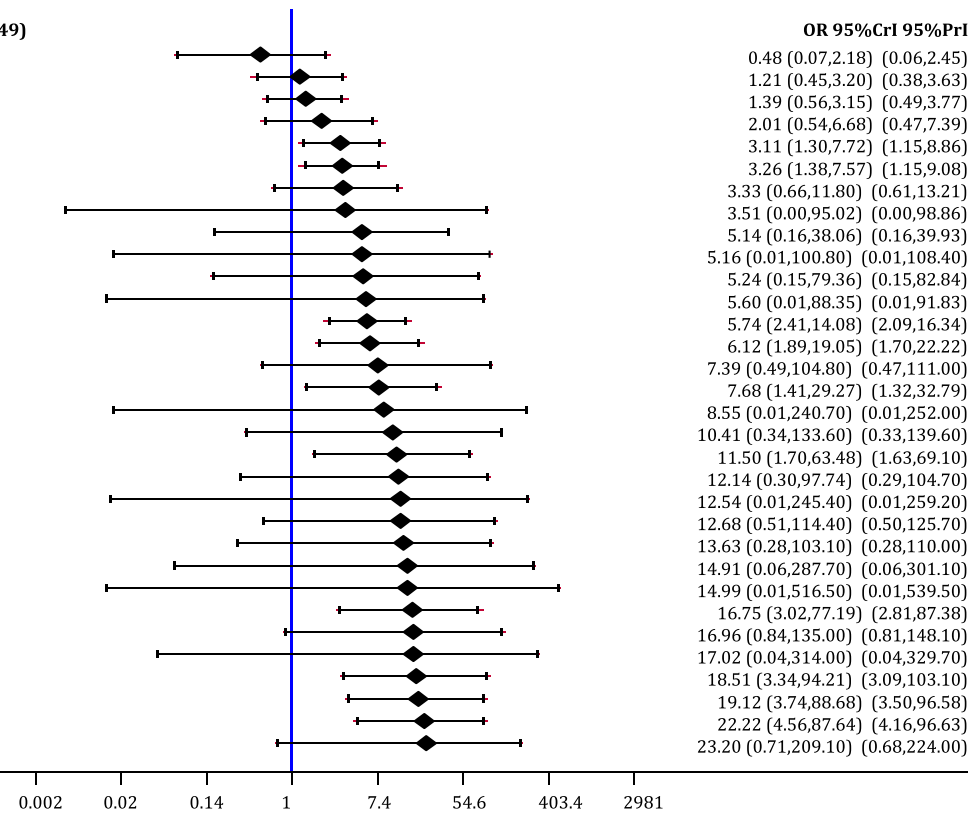

$5.14(0.16,38.06)(0.16,39.93)$

$5.24(0.15,79.36)(0.15,82.84)$

$5.60(0.01,88.35)(0.01,91.83)$

$5.74(2.41,14.08)(2.09,16.34)$

$6.12(1.89,19.05)(1.70,22.22)$

$7.39(0.49,104.80)(0.47,111.00)$

$7.68(1.41,29.27)(1.32 .32 .79)$

$8.55(0.01,240.70)(0.01,252.00)$

$10.41(0.34,133.60)(0.33,139.60)$

$11.50(1.70,63.48)(1.63,69.10)$

$11.50(1.70,63.48)(1.63,69.10)$

$12.14(0.30,97.74)(0.29,104.70)$

$12.54(0.01,245.40)(0.01,259.20)$

$12.68(0.51,114.40)(0.50,125.70)$

$13.63(0.28,103.10)(0.28,110.00)$

$14.91(0.06,287.70)(0.06,301.10)$

$14.99(0.01,516.50)(0.01,539.50)$

$16.75(3.02,77.19)(2.81,87.38)$

$16.96(0.84,135.00)(0.81,148.10)$

$17.02(0.04,314.00)(0.04,329.70)$

$18.51(3.34,94.21)(3.09,103.10)$

$19.12(3.74,88.68)(3.50,96.58)$

$22.22(4.56,87.64)(4.16,96.63)$ $23.20(0.71,209.10)(0.68,224.00)$ b

Active Treatment vs Control ( $n=777)$

Levet ( $\mathrm{n}=450$ )

Lamot ( $\mathrm{n}=1621)$

Pheno+Pheny $(\mathrm{n}=321)$

Pheno ( $n=1057)$

Carbam (n=99)

Topir $(\mathrm{n}=359)$

Oxcar ( $\mathrm{n}=198)$

Ethos+Pheny $(\mathrm{n}=4)$

Pheny+Valpro $(n=4)$

Pheny (n=932)

Valpro $(\mathrm{n}=802)$

Carbam+Pheno+Pheny $(\mathrm{n}=2)$

Clonaz ( $\mathrm{n}=34)$

Ethos+Pheno $(\mathrm{n}=3)$

Primid ( $\mathrm{n}=70)$

Carbam+Clonaz (n=7)

Pheny+Primid $(\mathrm{n}=7)$

Gabap (n=2)

Pheno+Primid (n=5)

Carbam+Pheno $(\mathrm{n}=26)$

Pheno+Valpro ( $\mathrm{n}=6$ )

Carbam+Pheny $(\mathrm{n}=8)$

Carbam+Valpro $(\mathrm{n}=21)$

Ethos ( $\mathrm{n}=10)$

Pheno+Pheny+Primid ( $\mathrm{n}=9$ )

Clobaz ( $\mathrm{n}=2)$
Club Foot

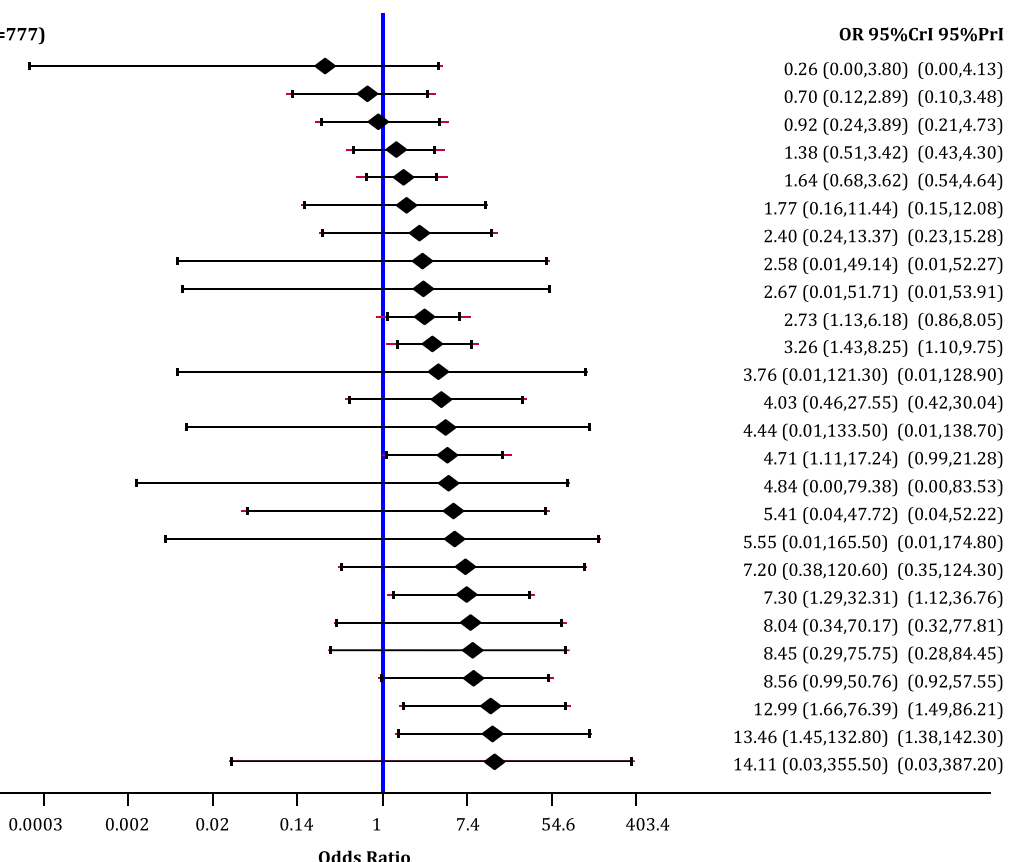

Fig. 7 Network meta-analysis forest plots for each treatment versus control. Each rhombus represents the summary treatment effect estimated in the network meta-analysis on the odds ratio (OR) scale. The black horizontal lines represent the credible intervals (Crl) for the summary treatment effects, and the red horizontal lines represent the corresponding predictive intervals (Prl). In the absence of heterogeneity, the Crls and Prls should be identical. An OR $>1$ suggests that control is safer, whereas an $\mathrm{OR}<1$ suggests that the comparator active treatment is safer. The vertical blue line corresponds to an $\mathrm{OR}=1$ (i.e., the treatment groups compared are equally safe). The total sample size (n) included in each treatment is also presented. a Cleft lip/palate (29 studies, 18,987 cases, 33 treatments). b Club foot (23 studies, 8836 cases 27 treatments). carbam carbamazepine, clobaz clobazam, clonaz clonazepam, ethos ethosuximide, gabap gabapentin, lamot lamotrigine, levet levetiracetam, oxcar oxcarbazepine, pheno phenobarbital, pheny phenytoin, primid primidone, topir topiramate, valpro val proate, vigab vigabatrin 


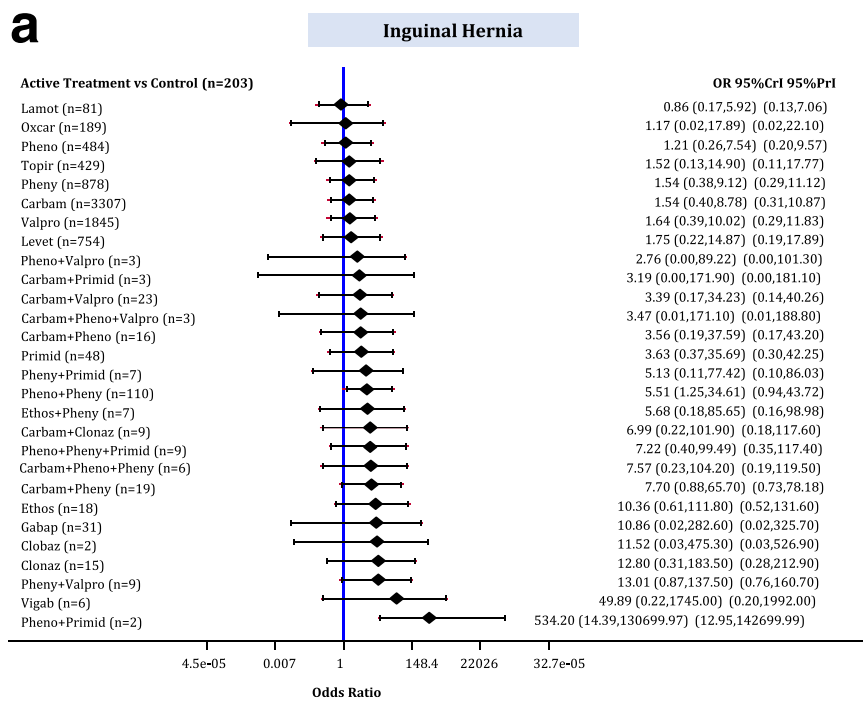

b

Undescended Testes

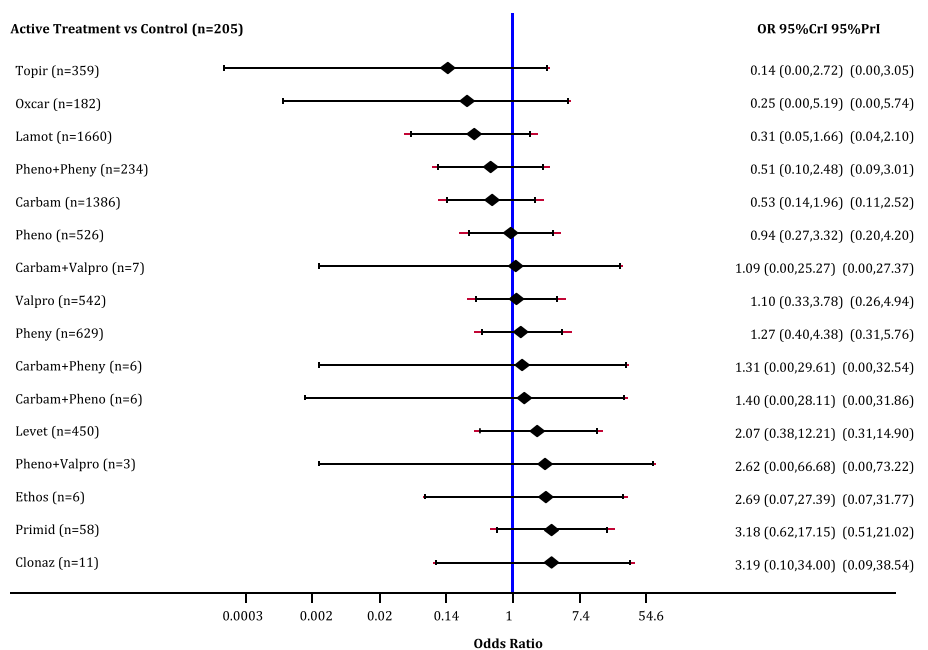

C

Active Treatment vs Control

Oxcar (n=35)

Clobaz+Oxcar (n=9)

Clonaz $+0 x$ car $(\mathrm{n}=9)$

Carbam+Clonaz $(\mathrm{n}=4)$

Pheno (n=127)

Clonaz (n=3)

Carbam+Pheno (n=30)

Pheny ( $\mathrm{n}=65)$

Carbam (n=164)

Primid ( $\mathrm{n}=9$ )

Carbam+Pheny (n=16)

Carbam+Pheno+Pheny (n=5)

Pheno+Valpro $(\mathrm{n}=20)$

Valpro (n=31)

Pheno+Pheny (n=56)

Carbam+Pheno+Valpro (n=3)
Any minor Congenital Malformations

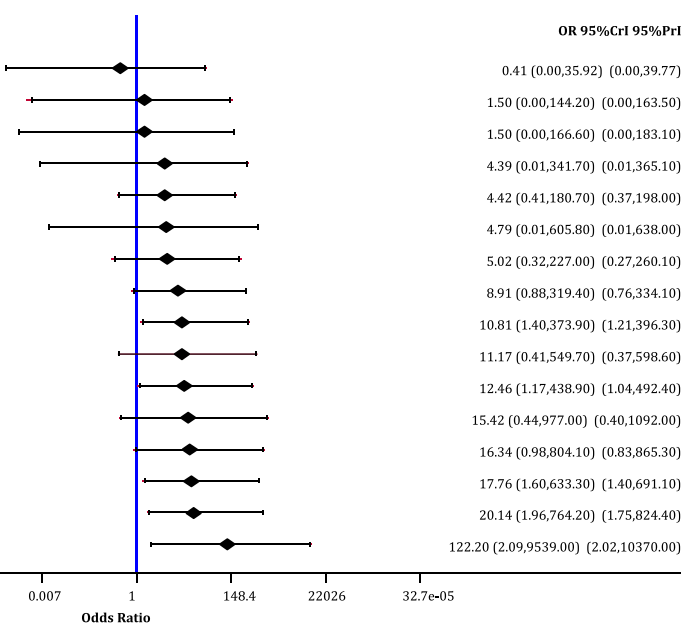

Fig. 8 (See legend on next page.) 
(See figure on previous page.)

Fig. 8 Network meta-analysis forest plots for each treatment versus control. Each rhombus represents the summary treatment effect estimated in the network meta-analysis on the odds ratio (OR) scale. The black horizontal lines represent the credible intervals (Crl) for the summary treatment effects, and the red horizontal lines represent the corresponding predictive intervals (Prl). In the absence of heterogeneity, the Crls and Prls should be identical. An OR $>1$ suggests that control is safer, whereas an $\mathrm{OR}<1$ suggests that the comparator active treatment is safer. The vertical blue line corresponds to an $\mathrm{OR}=1$ (i.e., the treatment groups compared are equally safe). The total sample size (n) included in each treatment is also presented. a Inguinal hernia (13 studies, 12,216 cases, 29 treatments). b Undescended testes (10 studies, 6270 cases, 17 treatments). c Minor congenital malformations (9 studies, 614 cases, 17 treatments). carbam carbamazepine, clobaz clobazam, clonaz clonazepam, ethos ethosuximide, gabap gabapentin, lamot lamotrigine, levet levetiracetam, oxcar oxcarbazepine, pheno phenobarbital, pheny phenytoin, primid primidone, topir topiramate, valpro valproate, vigab vigabatrin

Appendices J, L, and N). Phenobarbital plus phenytoin (OR, 5.51; 95\% CrI, 1.25-34.61) and phenobarbital plus primidone (OR, 534.20; 95\% CrI, $14.39-1.31 \times 10^{5}$ ) were associated with statistically significantly more cases developing inguinal hernia than control (Fig. 8a).

\section{Undescended testes}

The median baseline risk of undescended testes in the control group (no AED exposure) across all studies was 0.000 (interquartile range, $0.000-0.026$; Additional file 3: Appendix K). The NMA for undescended testes included 1 RCT, 1 case-control, and 8 cohort studies, 6270 boys, and 16 AEDs plus control, with $3 \%$ of comparisons reaching statistical significance (Additional file 3: Appendices J, $\mathrm{L}$, and N). Nothing was statistically significant versus control (Fig. 8b).

\section{Any minor CMs}

The median baseline risk of any minor $\mathrm{CM}$ in the control group (no AED exposure) across all studies was 0.000 (interquartile range, 0.000-0.000; Additional file 3: Appendix K). The NMA for minor CMs included 1 RCT and 8 studies, 614 cases, and 16 AEDs plus control, with $10 \%$ of comparisons reaching statistical significance (Additional file 3: Appendices J, L, and N). Carbamazepine (OR, 10.81; 95\% CrI, 1.40-373.90), carbamazepine pus phenytoin (OR, 12.46; 95\% CrI, 1.17-438.90), valproate (OR, 17.76; 95\% CrI, 1.60-633.30), phenobarbital plus phenytoin (OR, 20.14; 95\% CrI, 1.96-764.20), and carbamazepine plus phenobarbital plus valproate (OR, 122.20; 95\% CrI, 2.09-9539.00) were associated with statistically significantly more cases developing any minor CM than control (Fig. 8c).

\section{Discussion}

There is concern that most AEDs introduce the risk of abnormal or delayed physical development for infants who are exposed in utero. Our results show that, across major and minor $\mathrm{CM}$ outcomes, many AEDs were associated with higher risk of CMs than control. The monotherapies associated with statistically significant risk of CMs and prenatal harms compared to control across two or more NMAs were carbamazepine (overall major and minor $\mathrm{CMs}$ ), clobazam (prenatal growth retardation, preterm birth), ethosuximide (overall major $\mathrm{CM}$, cleft lip/palate, club foot), gabapentin (cardiac malformations, hypospadias), phenobarbital (overall major CM, prenatal growth retardation, cleft lip/palate), phenytoin (overall major CM, cleft lip/palate, club foot), topiramate (overall major CM, combined fetal losses, prenatal growth retardation, cleft lip/palate), and valproate (overall major and minor CMs, combined fetal losses, hypospadias, cleft lip/palate, club foot). Of these, only topiramate and gabapentin are newer generation AEDs. Gabapentin lacked sufficient evidence to reach statistical significance in overall major CM, and had an overall risk of malformations equivalent to control. This finding may be due to the inclusion of major malformations that were detected at birth only, which may decrease the possibility that all cardiac malformations were identified, especially those that can be detected later in childhood (or adulthood). Our results suggest that there is a significant association between topiramate and increased combined fetal losses. However, the treatment effect of topiramate versus control could only be estimated indirectly with high uncertainty. In the network, topiramate was informed by a single, small, five-arm cohort study [42], with only two patients exposed in topiramate (total sample size, $n=25$ ) and low methodological quality regarding the comparability of cohorts and adequacy of follow-up. The following newer generation AEDs were not associated with statistically significant risks to physical development compared to control: lamotrigine $(n=6290)$, levetiracetam $(n=1015)$, oxcarbazepine $(n=372)$, and vigabatrin $(n=23)$. However, this does not mean that these agents are not harmful to the offspring of mothers administered these agents (i.e., risks have not been ruled out). Overall, the newer AED agents, including levetiracetam and lamotrigine, were associated with lower risk of overall major CMs and CMs by specific type; however, data from more patients were available for lamotrigine than levetiracetam (6290 versus 1015 total infants, respectively), thereby providing greater confidence in lamotrigine's safety profile. Further, lamotrigine ranked as the second safest monotherapy for prenatal growth retardation, and was comparable to control for preterm birth. Phenobarbital was the AED monotherapy with the lowest risk of fetal loss, whereas 
phenytoin was the monotherapy associated with the lowest risk of impaired prenatal growth retardation. Vigabatrin and oxcarbazepine were the least likely monotherapies to increase the risk for preterm birth; however, vigabatrin included only 13 infants compared to the 1045 infants in oxcarbazepine, which contributed to the lower precision in the estimation of vigabatrin's SUCRA curve value (Fig. 2 and Additional file 3: Appen$\operatorname{dix} \mathrm{N})$. While gabapentin and clonazepam were ranked as moderately safe, more data are needed to elucidate their potential teratogenicity (329 and 375 infants in total, respectively). Across all outcomes, the following polytherapies were associated with both statistically significant CMs and prenatal harms compared with control across two or more of our NMAs: phenobarbital plus phenytoin, carbamazepine plus phenobarbital, carbamazepine plus phenytoin, phenobarbital plus valproate, phenytoin plus primidone, phenytoin plus valproate, carbamazepine plus valproate, carbamazepine plus clonazepam, phenobarbital plus phenytoin plus primidone, and phenobarbital plus primidone. There is insufficient evidence to make any conclusions regarding polytherapy with newer generation AEDs due to a lack of studies reporting these combinations.

Our study has several strengths. First, we followed the guidelines in the Cochrane Handbook for systematic reviews and ISPOR for NMAs [14], and we reported our findings according to recommendations included the PRISMA-NMA statement [15]. Second, using NMA methods, we were able to compare treatments that have not been compared in previous head-to-head studies, as well as provide a hierarchy of the treatments according to their safety (through the SUCRA curves) [7]. In addition, the complexity of the evidence identified in our systematic review is, in contrast to a pairwise metaanalysis model, properly accounted in a NMA model, which models within-trial correlations induced by the multi-arm studies [43]. Third, our study results are based on a larger number of studies compared to previous knowledge syntheses [7]. A previous systematic review [7] including 59 studies and a total of 65,553 pregnant women examined the risk of malformations in women with epilepsy and showed that the most common were cardiac malformations. The number of pregnancies in this review was higher than our systematic review because of the inclusion of studies that did not analyze the risk by AED and used unspecified polytherapy, which could not be included in our NMA. In contrast to this review, our study assesses each AED separately for both overall and specific malformations, and hence our results are not directly comparable to this review. Fourth, we accounted for the different study designs by applying the Schmitz et al. [33] approach. In this three-level hierarchical model we considered two different sources of evidence, i.e., the observational studies, including cohort and case-control studies, and the RCTs. To account for the potential differences between cohort studies and case-control studies, as the approaches of these two methodologies vary, we conducted a sensitivity analysis restricting to cohort studies $(k=75)$ for the primary outcome, which included all study designs and the greatest number of case-control studies $(k=2)$ and RCTs $(k=1)$. As expected, since the majority of the included study designs were cohort studies, all approaches suggested comparable results. To the best of our knowledge, our study was the first to compare and rank the safety of AEDs using the SUCRA curves and rank-heat plots [36, 37].

Our study has some limitations worth noting. First, we did not incorporate differences in drug dosages of the AEDs because this information was rarely reported across the included studies, although a dose-response relationship has been observed for these agents. For instance, a potential modification of the estimated treatment effects may occur if the doses vary considerably across treatment indications, and accounting for the fact that certain AEDs were more widely utilized in other conditions, while some AEDs are almost exclusively used for epilepsy. Second, the paucity of available data is a limitation; many polytherapies were informed by only a few studies and patients, and many studies included zero events in all arms for the specific CMs and were excluded from those analyses. This impacted the treatment group risk across studies; for example, the median risk of the major congenital anomalies per treatment ranged between $0 \%$ and $24 \%$. The lack of adequate knowledge of risks for multiple AEDs impacts the NMA results. This affected the SUCRA estimates, which showed several polytherapies with high OR estimates, but with extremely wide CrIs. For example, in overall major CMs, nine polytherapies had SUCRA curve estimates above $74 \%$, but these all had wide CrIs (95\% CrI with shorter length, 28-96\%; 95\% CrI with wider length, 0-100\%) potentially due to the small number of patients (range, 3-21) and studies (range, 1-2) informing these interventions (Additional file 3: Appendix K). Indeed, a simulation study [44] assessing the ranking probability for a treatment of being the best in NMA with a different number of studies per comparison, suggested that the probability of being the best may be biased in favor of treatments with a smaller number of studies. Additionally, another study indicated that the SUCRA curve values might be unreliable [45]. As such, our SUCRA curve values need to be interpreted in conjunction with the ORs and 95\% CrIs. Third, quality of reporting of the identified observational studies may have introduced bias [46]; $81 \%$ did not control for important cofounders, such as maternal age and epilepsy type and severity, and 59\% had large attrition rates. 
Further, some registries measured CMs and there is a risk that may not have consistently collected data on different types of fetal losses (e.g., stillbirths). However, studies were internally consistent across arms with respect to what was reported. The inclusion of observational studies adds on the evaluation of the safety profile of AED treatments and offers the opportunity to generalize evidence. Fourth, despite no evidence of inconsistency, the assessment of transitivity for most treatment effect modifiers suggested that there was an imbalance in the different levels of quality appraisal across treatment comparisons and most outcomes, which may affect NMA results. A possible approach to address this in a future study would be the use of individual patient data in NMA, to allow for adjustment of the relative treatment effects from the observational studies utilizing patient level covariates. This would also aid decision-making to allow tailoring management to individual patient characteristics [47]. Fifth, although adjusted funnel plots suggested no evidence of publication bias and small-study effects, asymmetry may have been masked given several studies compared multiple arms. To reduce the majority of correlations induced by multi-arm studies, we plotted data points corresponding to the study-specific basic parameters. Additionally, babies born every day are exposed to AEDs and although we searched extensively for grey (i.e., difficult to locate or unpublished) literature, we may have missed unpublished data relevant to our research question. Sixth, the strength of evidence in most NMAs may be low due to the small number of studies compared to the number of treatments included in each network. However, the predictive intervals suggested that our results are robust, overall. Seventh, we combined data across study designs to determine how AEDs behave in the 'real world'. However, this may have introduced heterogeneity in our analyses. We used the naive approach and the Schmitz et al. [33] model to combine different study designs, as well as sensitivity analyses on observational and cohort studies separately, and all approaches suggested similar results. Although RCTs are considered to be the gold standard of evidence, we included observational studies in our analyses due to the dearth of available RCTs.

It should be highlighted that, although some of the individual malformations in this review exceeded the number of pregnancies yielding malformations, the unit of analysis in our study was the number of infants with a malformation at birth. Therefore, discussion of the prevalence of multiple malformations would be beyond the scope of the current article. Future studies should assess safety and effectiveness of AEDs for pregnant women considering factors that could affect the results, such as alcohol and folic acid use. Observational studies should follow the STROBE guidance to improve the quality of reporting [48]. Despite recent large-scale registries evaluating rare harms [28, 49-52], more evidence is required to conclude which polytherapy is the safest, especially for the newer-generation AEDs, and to allow better tailoring for patients with different characteristics such as history of alcohol use. Registries should aim to include a suitable control group and collect information on potential confounders to inform which agents are the safest.

\section{Conclusions}

The large volume of evidence in this analysis suggests that the newer generation AEDs, lamotrigine and levetiracetam, were not associated with statistically significant increased risks to CMs compared to control, and were statistically significantly less likely to be associated with children experiencing cardiac malformations than control. In contrast, the risk of malformations was increased for ethosuximide, valproate, topiramate, phenobarbital, phenytoin, carbamazepine, and 11 polytherapies. Additionally, a significant association between topiramate and increased combined fetal losses was identified. However, caution is needed, as the overall low quality of the research available on this subject limits what can be definitively concluded and AEDs may be potentially harmful to infants and children exposed in utero. Counselling is advised concerning teratogenic risks when the prescription is first written for a woman of childbearing potential and before women continue with these agents when considering pregnancy, such as switching from polytherapy to monotherapies with evidence of lower risk and avoiding AEDs, such as valproate, that are consistently associated with CMs. These decisions must be balanced against the need for seizure control.

\section{Additional files}

\section{Abbreviations}

AED: anti-epileptic drugs; CM: congenital malformations; Crl: credible interval; NMA: network meta-analysis; OR: odd ratios; RCT: randomized clinical trials; SUCRA: surface under the cumulative ranking curve 


\section{Acknowledgements}

We were commissioned to conduct this research for policymakers from Health Canada through the Canadian Institutes of Health Research Drug Safety and Effectiveness Network.

We thank Dr. David Moher for providing his feedback on our protocol. We thank Dr. Laure Perrier for conducting the literature searches, Becky Skidmore for peer-reviewing the MEDLINE search, and Alissa Epworth for obtaining the full-text articles. We thank Alistair Scott, Wing Hui, and Geetha Sanmugalingham for screening some of the citations and/or abstracting some of the data for a few of the included studies, Misty Pratt and Mona Ghannad for helping scan reference lists, and Ana Guzman, Susan Le, and Inthuja Selvaratnam for contacting authors and formatting the manuscript. We also thank Dr. Anick Bérard, Dr. Sonia Hernandez-Diaz, Dr. Pernille E. Jacobsen, Dr. Silvia Kochen, Professor G. Mawer, Ms. Ditte Mølgaard-Nielsen, Dr. S.V. Thomas, and Dr. Gyri Veiby for providing us with clarifications and/or additional data.

\section{Funding}

This systematic review was funded by the Canadian Institutes for Health Research/Drug Safety and Effectiveness Network (CIHR/DSEN). AAV is funded by the Banting Postdoctoral Fellowship Program from the CIHR. SES is funded by a Tier 1 Canada Research Chair in Knowledge Translation. BH is funded by a CIHR/DSEN New Investigator Award in Knowledge Synthesis. $\mathrm{BRH}$ receives funding from the Alberta Heritage Foundation for Medical Research. ACT is funded by a Tier 2 Canada Research Chair in Knowledge Synthesis. The funder had no role in the design and conduct of the study; collection, management, analysis, and interpretation of the data; preparation, review, or approval of the manuscript; or decision to submit the manuscript for publication.

\section{Availability of data and materials}

All datasets generated and/or analyzed during the current study are available from the corresponding author on reasonable request.

\section{Authors' contributions}

AAV analyzed the data, interpreted the results, and helped write the manuscript. ACT and SES conceived and designed the study, helped obtain funding, interpreted the results, and wrote sections of the manuscript. EC and PR coordinated the review, screened citations and full-text articles, abstracted data, appraised quality, resolved discrepancies, contacted authors, and edited the manuscript. CS provided methodological support, screened citations and full-text articles, and edited the manuscript. RK, ER, FY, JDS, KT, and HM screened citations and full-text articles, abstracted data, and/or appraised quality. BH, BRH and YF helped conceive the study and edited the manuscript. All authors read and approved the final manuscript. All authors, external and internal, had full access to all of the data (including statistical reports and tables) in the study and can take responsibility for the integrity of the data and the accuracy of the data analysis.

\section{Competing interests}

Dr. Andrea Tricco is an associate editor for BMC Medicine but was not involved with the peer-review process/decision to publish.

\section{Consent for publication}

Not applicable.

\section{Ethics approval and consent to participate}

Not applicable.

\section{Publisher's Note}

Springer Nature remains neutral with regard to jurisdictional claims in published maps and institutional affiliations.

\section{Author details}

${ }^{1}$ Knowledge Translation Program, Li Ka Shing Knowledge Institute, St. Michael's Hospital, 209 Victoria Street, East Building, Toronto, Ontario M5B 1W8, Canada. ${ }^{2}$ Department of Geriatric Medicine, University of Toronto, 27 King's College Circle, Toronto, Ontario M5S 1A1, Canada. ${ }^{3}$ The Hospital for Sick Children, 555 University Avenue, Toronto, Ontario M5G 1X8, Canada. ${ }^{4}$ Department of Paediatrics, University of Toronto, 172 St. George Street, Toronto, Ontario M5R 0A3, Canada. ${ }^{5}$ Department of Pharmacology and Toxicology, University of Toronto, Medical Sciences Building, Room 4207, 1
King's College Circle, Toronto, Ontario M5S 1A8, Canada. Institute for Health Policy Management \& Evaluation, University of Toronto, 4th Floor, 155 College Street, Toronto, Ontario M5T 3M6, Canada. 'School of Epidemiology, Public Health and Preventive Medicine, Faculty of Medicine, University of Ottawa, Roger-Guindon Building, 451 Smyth Road, Ottawa, Ontario K1H 8M5, Canada. ${ }^{8}$ Clinical Epidemiology Program, Ottawa Hospital Research Institute, The Ottawa Hospital, 501 Smyth Road, Ottawa, Ontario K1H 8L6, Canada. ${ }^{9}$ Institute of Clinical and Evaluative Sciences (ICES uOttawa), 1053 Carling Ave, Ottawa, Ontario K1Y 4E9, Canada. ${ }^{10}$ Ottawa Hospital Research Institute, Center for Practice Changing Research, The Ottawa Hospital - General Campus, 501 Smyth Road, PO Box 201B, Ottawa, Ontario K1H 8L6, Canada. ${ }^{11}$ Departments of Medicine and Community Health Sciences, University of Calgary, TRW Building, 3rd Floor, 3280 Hospital Drive NW, Calgary, Alberta T2N 4Z6, Canada. ${ }^{12}$ Epidemiology Division, Dalla Lana School of Public Health, University of Toronto, 6th Floor, 155 College Street, Toronto, Ontario M5T 3M7, Canada.

Received: 14 November 2016 Accepted: 28 March 2017

Published online: 05 May 2017

\section{References}

1. Hauser W, Hesdorffer D. Epilepsy: Frequency, Causes and Consequences. New York: Demos Medical Pub; 1990.

2. Wiebe S, Bellhouse DR, Fallahay C, Eliasziw M. Burden of epilepsy: the Ontario Health Survey. Can J Neurol Sci. 1999;26(4):263-70.

3. Morrell MJ. Epilepsy in women. Am Fam Physician. 2002;66(8):1489-94.

4. Zahn CA, Morrell MJ, Collins SD, Labiner DM, Yerby MS. Management issues for women with epilepsy: a review of the literature. Neurology. 1998;51(4):949-56.

5. Spina E, Perugi G. Antiepileptic drugs: indications other than epilepsy. Epileptic Disord. 2004;6(2):57-75

6. Harden CL, Pennell PB, Koppel BS, Hovinga CA, Gidal B, Meador KJ, et al. Management issues for women with epilepsy-focus on pregnancy (an evidence-based review): III. vitamin K, folic acid, blood levels, and breastfeeding: report of the quality standards subcommittee and therapeutics and technology assessment subcommittee of the American Academy of Neurology and the American Epilepsy Society. Epilepsia. 2009;50(5):1247-55.

7. Meador K, Reynolds MW, Crean S, Fahrbach K, Probst C. Pregnancy outcomes in women with epilepsy: a systematic review and meta-analysis of published pregnancy registries and cohorts. Epilepsy Res. 2008;81(1):1-13.

8. Samren EB, van Duijn CM, Koch S, Hiilesmaa VK, Klepel H, Bardy AH, et al. Maternal use of antiepileptic drugs and the risk of major congenital malformations: a joint European prospective study of human teratogenesis associated with maternal epilepsy. Epilepsia. 1997;38(9):981-90.

9. Janz D, Fuchs U. Are anti-epileptic drugs harmful during pregnancy? Dtsch Med Wochenschr. 1964;89:241-8.

10. Meadow SR. Anticonvulsant drugs and congenital abnormalities. Lancet. 1968:2(7581):1296.

11. Shorvon SD. Drug treatment of epilepsy in the century of the ILAE: the second 50 years, 1959-2009. Epilepsia. 2009;50 Suppl 3:93-130.

12. Johannessen Landmark C, Patsalos PN. Drug interactions involving the new second- and third-generation antiepileptic drugs. Expert Rev Neurother. 2010;10(1):119-40

13. Tricco AC, Cogo E, Veroniki AA, Soobiah C, Hutton B, Hemmelgarn BR, et al. Comparative safety of anti-epileptic drugs among infants and children exposed in utero or during breastfeeding: protocol for a systematic review and network meta-analysis. Syst Rev. 2014;3:68.

14. Jansen JP, Trikalinos T, Cappelleri JC, Daw J, Andes S, Eldessouki R, et al. Indirect treatment comparison/network meta-analysis study questionnaire to assess relevance and credibility to inform health care decision making: an ISPOR-AMCP-NPC Good Practice Task Force report. Value Health. 2014;17(2):157-73

15. Hutton B, Salanti G, Caldwell DM, Chaimani A, Schmid CH, Cameron C, et al. The PRISMA extension statement for reporting of systematic reviews incorporating network meta-analyses of health care interventions: checklist and explanations. Ann Intern Med. 2015;162(11):777-84.

16. Harden C, Thomas S, Tomson T. Epilepsy in Women. West Sussex, UK: Wiley-Blackwell; 2013.

17. Sampson M, McGowan J, Cogo E, Grimshaw J, Moher D, Lefebvre C. An evidence-based practice guideline for the peer review of electronic search strategies. J Clin Epidemiol. 2009;62(9):944-52. 
18. Higgins JP, Altman DG, Gotzsche PC, Juni P, Moher D, Oxman AD, et al. The Cochrane Collaboration's tool for assessing risk of bias in randomised trials. BMJ. 2011;343:d5928.

19. The Newcastle-Ottawa Scale (NOS) for assessing the quality of nonrandomised studies in meta-analyses. http://www.ohri.ca/programs/ clinical_epidemiology/oxford.asp. Accessed 6 Apr 2017.

20. Chaimani A, Higgins JP, Mavridis D, Spyridonos P, Salanti G. Graphical tools for network meta-analysis in STATA. PLoS One. 2013;8(10):e76654.

21. LU G, Ades AE. Combination of direct and indirect evidence in mixed treatment comparisons. Stat Med. 2004;23(20):3105-24.

22. Jansen JP, Naci H. Is network meta-analysis as valid as standard pairwise meta-analysis? It all depends on the distribution of effect modifiers. BMC Med. 2013;11:159.

23. White IR, Barrett JK, Jackson D, Higgins JPT. Consistency and inconsistency in network meta-analysis: model estimation using multivariate meta-regression. Res Synth Methods. 2012;3(2):111-25.

24. Higgins JP, Jackson D, Barrett JK, Lu G, Ades AE, White IR. Consistency and inconsistency in network meta-analysis: concepts and models for multi-arm studies. Res Synth Methods. 2012;3(2):98-110.

25. Song F, Altman DG, Glenny AM, Deeks JJ. Validity of indirect comparison for estimating efficacy of competing interventions: empirical evidence from published meta-analyses. BMJ. 2003;326(7387):472.

26. Veroniki AA, Vasiliadis HS, Higgins JP, Salanti G. Evaluation of inconsistency in networks of interventions. Int J Epidemiol. 2013;42(1):332-45.

27. Higgins JPT, Green S (editors). Cochrane Handbook for Systematic Reviews of Interventions Version 5.1.0 [updated March 2011]. The Cochrane Collaboration, 2011. www.handbook.cochrane.org. Accessed 6 Apr 2017.

28. Tomson T, Battino D, Bonizzoni E, Craig J, Lindhout D, Sabers A, et al. Dose-dependent risk of malformations with antiepileptic drugs: an analysis of data from the EURAP epilepsy and pregnancy registry. Lancet Neurol. 2011;10(7):609-17.

29. Tomson T, Battino D, Bonizzoni E, Craig J, Lindhout D, Perucca E, et al. Dose-dependent teratogenicity of valproate in mono- and polytherapy: an observational study. Neurology. 2015;85(10):866-72.

30. Vajda FJ, O'Brien TJ, Lander CM, Graham J, Eadie MJ. The teratogenicity of the newer antiepileptic drugs - an update. Acta Neurol Scand. 2014;130(4):234-8.

31. Martinez Ferri M, Pena Mayor P, Perez Lopez-Fraile I, Castro Vilanova MD, Escartin Siquier A, Martin Moro M, et al. Malformations and fetal death in the Spanish antiepileptic drug and pregnancy registry: results at 6 years. Neurologia. 2009;24(6):360-5.

32. Kochen S, Salera C, Seni J. Pregnant women with epilepsy in a developing country. Open Neurol J. 2011;5:63-7.

33. Schmitz $\mathrm{S}$, Adams $\mathrm{R}$, Walsh C. Incorporating data from various trial designs into a mixed treatment comparison model. Stat Med. 2013;32(17):2935-49.

34. Spiegelhalter DJ, Best NG, Carlin BP, Van Der Linde A. Bayesian measures of model complexity and fit. J R Stat Soc Ser B Stat Methodol. 2002;64(4):583-639.

35. Welton NJ, Sutton AJ, Cooper N, Abrams KR, Ades A. Evidence synthesis for decision making in healthcare. New York: Wiley; 2012.

36. Salanti G, Ades AE, loannidis JP. Graphical methods and numerical summaries for presenting results from multiple-treatment meta-analysis: an overview and tutorial. J Clin Epidemiol. 2011;64(2):163-71.

37. Veroniki AA, Straus SE, Fyraridis A, Tricco AC. The rank-heat plot is a novel way to present the results from a network meta-analysis including multiple outcomes. J Clin Epidemiol. 2016;76:193-9.

38. Lunn D, Spiegelhalter D, Thomas A, Best N. The BUGS project: evolution, critique and future directions. Stat Med. 2009;28(25):3049-67.

39. Palmer T, Sterne J. Meta-Analysis in Stata: An Updated Collection from the Stata Journal. Texas: Stata Press; 2016.

40. Higgins JP, Thompson SG, Spiegelhalter DJ. A re-evaluation of random-effects meta-analysis. J R Stat Soc Ser A Stat Soc. 2009;172(1):137-59.

41. Riley RD, Higgins JP, Deeks JJ. Interpretation of random effects metaanalyses. BMJ. 2011;342:d549.

42. Babic M, Jovic N. Postnatal concerns in children born to women with juvenile myoclonic epilepsy. In: European Congress on Epileptology, vol. 55. Stockholm: Epilepsia; 2014. p. 128.

43. Franchini AJ, Dias S, Ades AE, Jansen JP, Welton NJ. Accounting for correlation in network meta-analysis with multi-arm trials. Res Synth Methods. 2012;3(2):142-60.
44. Kibret T, Richer D, Beyene J. Bias in identification of the best treatment in a Bayesian network meta-analysis for binary outcome: a simulation study. Clin Epidemiol. 2014;6:451-60.

45. Trinquart L, Attiche N, Bafeta A, Porcher R, Ravaud P. Uncertainty in treatment rankings: reanalysis of network meta-analyses of randomized trials. Ann Intern Med. 2016;164(10):666-73.

46. Cameron C, Fireman B, Hutton B, Clifford T, Coyle D, Wells G, et al. Network meta-analysis incorporating randomized controlled trials and non-randomized comparative cohort studies for assessing the safety and effectiveness of medical treatments: challenges and opportunities. Syst Rev. 2015:4:147.

47. Veroniki AA, Straus SE, Soobiah C, Elliott MJ, Tricco AC. A scoping review of indirect comparison methods and applications using individual patient data. BMC Med Res Methodol. 2016;16(1):47.

48. von Elm E, Altman DG, Egger M, Pocock SJ, Gotzsche PC, Vandenbroucke JP. The Strengthening the Reporting of Observational Studies in Epidemiology (STROBE) statement: guidelines for reporting observational studies. PLoS Med. 2007;4(10):e296.

49. Campbell E, Kennedy F, Russell A, Smithson WH, Parsons L, Morrison PJ, et al. Malformation risks of antiepileptic drug monotherapies in pregnancy: updated results from the UK and Ireland Epilepsy and Pregnancy Registers. J Neurol Neurosurg Psychiatry. 2014;85(9):1029-34.

50. Hernandez-Diaz S, Smith CR, Shen A, Mittendorf R, Hauser WA, Yerby M et al. Comparative safety of antiepileptic drugs during pregnancy. Neurology. 2012;78(21):1692-9.

51. Kallen B, Borg N, Reis M. The use of central nervous system active drugs during pregnancy. Pharmaceuticals (Basel). 2013;6(10):1221-86.

52. Veiby G, Daltveit AK, Engelsen BA, Gilhus NE. Fetal growth restriction and birth defects with newer and older antiepileptic drugs during pregnancy. J Neurol. 2014;261(3):579-88.

\section{Submit your next manuscript to BioMed Central and we will help you at every step:}

- We accept pre-submission inquiries

- Our selector tool helps you to find the most relevant journal

- We provide round the clock customer support

- Convenient online submission

- Thorough peer review

- Inclusion in PubMed and all major indexing services

- Maximum visibility for your research

Submit your manuscript at www.biomedcentral.com/submit 